\title{
Adult dyslexic readers benefit less from visual input during audiovisual speech processing: fMRI evidence
}

\author{
Ana A. Francisco ${ }^{\mathrm{a}, *}$, Atsuko Takashima ${ }^{\mathrm{b}}$, James M. McQueen ${ }^{\mathrm{b}}$, Mark van den Bunt ${ }^{\mathrm{a}}$, \\ Alexandra Jesse $^{\mathrm{c}}$, Margriet A. Groen ${ }^{\mathrm{a}}$ \\ a Behavioural Science Institute, Radboud University, Postbus 9104, 6500 HE Nijmegen, The Netherlands \\ ${ }^{\mathrm{b}}$ Donders Institute for Brain, Cognition and Behaviour, Radboud University, Max Planck Institute for Psycholinguistics, Nijmegen, The Netherlands \\ ${ }^{c}$ Department of Psychological and Brain Sciences, University of Massachusetts, USA
}

\section{A R T I C L E I N F O}

\section{Keywords:}

FMRI

Dyslexia

Audiovisual speech perception

Visual speech

\begin{abstract}
A B S T R A C T
The aim of the present fMRI study was to investigate whether typical and dyslexic adult readers differed in the neural correlates of audiovisual speech processing. We tested for Blood Oxygen-Level Dependent (BOLD) activity differences between these two groups in a 1-back task, as they processed written (word, illegal consonant strings) and spoken (auditory, visual and audiovisual) stimuli. When processing written stimuli, dyslexic readers showed reduced activity in the supramarginal gyrus, a region suggested to play an important role in phonological processing, but only when they processed strings of consonants, not when they read words. During the speech perception tasks, dyslexic readers were only slower than typical readers in their behavioral responses in the visual speech condition. Additionally, dyslexic readers presented reduced neural activation in the auditory, the visual, and the audiovisual speech conditions. The groups also differed in terms of superadditivity, with dyslexic readers showing decreased neural activation in the regions of interest. An additional analysis focusing on visionrelated processing during the audiovisual condition showed diminished activation for the dyslexic readers in a fusiform gyrus cluster. Our results thus suggest that there are differences in audiovisual speech processing between dyslexic and normal readers. These differences might be explained by difficulties in processing the unisensory components of audiovisual speech, more specifically, dyslexic readers may benefit less from visual information during audiovisual speech processing than typical readers. Given that visual speech processing supports the development of phonological skills fundamental in reading, differences in processing of visual speech could contribute to differences in reading ability between typical and dyslexic readers.
\end{abstract}

\section{Introduction}

The development of adequate grapheme-phoneme associations is pivotal in reading. Neuroimaging studies consistently report inefficient processing of grapheme-phoneme associations in dyslexic readers (e.g., Blau et al., 2009). Given that such associations are fundamentally audiovisual in nature, an audiovisual deficit might underlie reading impairment in dyslexia (Blomert, 2011; Hahn et al., 2014). But such a deficit might be broader, that is, it might not be restricted to associations between letters and speech sounds. Dyslexic readers, when compared to typical readers, might therefore also process differently other types of audiovisual material, such as audiovisual speech. In the present functional Magnetic Resonance Imaging (fMRI) study we investigated whether dyslexic and typical adult readers differed in the neural correlates of audiovisual speech processing.

Dyslexia, characterized by severe reading and/or spelling impairments, represents a persistent condition: Individuals with a childhood diagnosis of dyslexia still manifest reading impairment in adulthood (e.g. Bekebrede et al., 2010; Elbro et al., 1994; van Bergen et al., 2014). Efficient learning and storing of the mappings between graphemes and phonemes is crucial for the development of an adequate reading level (Ehri, 1998). In line with this, the inadequacy of letter-sound mappings seems to be a main cause of reading impairment in dyslexia (Vellutino et al., 2004). Neuroimaging studies have consistently reported deficient processing of letter-sound associations in dyslexia. When presented with unisensory (auditory and visual) and audiovisual grapheme-

* Corresponding author. Present address: Cognitive Neurophysiology Laboratory, Department of Pediatrics, Albert Einstein College of Medicine, Van Etten Building, Room 1C-3B, 1225 Morris Park Avenue, Bronx, NY 10461, USA.

E-mail addresses: claraafrancisco@gmail.com (A.A. Francisco), atsuko.takashima@donders.ru.nl (A. Takashima), j.mcqueen@donders.ru.nl (J.M. McQueen), m.vandenbunt@pwo.ru.nl (M. van den Bunt), ajesse@psych.umass.edu (A. Jesse), m.groen@pwo.ru.nl (M.A. Groen). 
phoneme combinations, adult dyslexic readers showed reduced activation in both unisensory and multisensory regions: superior temporal cortex (Blau et al., 2009; Kast et al., 2011; Kronschnabel et al., 2014), supramarginal gyrus (Kast et al., 2011), left inferior frontal and angular gyri (Kronschnabel et al., 2014), and inferior temporal cortex (Kronschnabel et al., 2014).

While the focus of these studies has been on print-speech associations, there is behavioral evidence suggesting that the audiovisual deficit in dyslexia might be broader, namely that dyslexic readers also differ from typical readers when processing other types of audiovisual material, such as non-speech events (tones and circles; e.g., Hairston et al., 2005) and speech (e.g., Francisco et al., 2017; Hayes et al., 2003; Norrix et al., 2006; Ramirez and Mann, 2005; van Laarhoven et al., 2016) (but see Baart et al., 2012; de Gelder and Vroomen, 1998; Groen and Jesse, 2013 for reports of intact audiovisual processing in dyslexia). These results may thus suggest a broader audiovisual deficit that impairs not only the formation of (audiovisual) grapheme-phoneme associations, but also the processing of speech and non-speech audiovisual stimuli. The hindered formation of grapheme-phoneme associations, fundamental for reading, could result in impaired reading. Inadequate representations could create ambiguity in the correspondences between graphemes and phonemes (Wallace and Stevenson, 2014), which could result in slower decoding and reduced accuracy while reading (Hairston et al., 2005). The study of the neural correlates of audiovisual speech processing in dyslexia is therefore useful for two reasons. First, the current neuroimaging literature has focused almost exclusively on grapheme-phoneme associations. Studying audiovisual speech might offer additional support to the existing behavioral literature suggesting a broader audiovisual deficit in dyslexia. Second, it makes it possible to test the hypothesis of an audiovisual deficit in dyslexia without probing letter-sound associations, dyslexic readers' direct area of difficulty, while still using ecologically valid stimuli.

In typical readers, a number of brain regions have been repeatedly implicated in audiovisual speech processing. These regions include high-level associative areas such as the superior temporal gyrus (including the planum temporale) (e.g., Beauchamp et al., 2004a; Beauchamp et al., 2010; Calvert et al., 2000; Stevenson et al., 2010; Stevenson et al., 2011) and the supramarginal gyrus (e.g., Skipper et al., 2005), as well as other more primary sensory regions such as Heschl's gyrus (e.g., Calvert et al., 1999; Callan et al., 2003; Möttönen et al., 2004; Pekkola et al., 2005) and the superior temporal gyrus (e.g., Beauchamp et al., 2004a, 2010; Calvert et al., 2000) for auditory information, and the middle temporal gyrus (e.g., Callan et al., 2003; Calvert et al., 1999; Calvert and Campbell, 2003) and the fusiform gyrus (e.g., Calvert and Campbell, 2003; Macaluso et al., 2004; Stevenson et al., 2010; Wyk et al., 2010) for visual information processing.

Some studies have shown that the regions involved in multisensory processing exhibit enhanced responses to audiovisual stimuli, when compared to the sum of the responses to unisensory auditory and visual stimuli (Giard and Peronnet, 1999; Calvert et al., 2000, 2001; Macaluso et al., 2000; Bushara et al., 2001; Klucharev et al., 2003; Wright et al., 2003; Molholm et al., 2004). This pattern is referred to as superadditivity and has been suggested to reflect basic sensory features of audiovisual integration (Kronschnabel et al., 2014). Interestingly, superadditivity seems to relate to behavioral measures of audiovisual integration: Individuals who benefit from audiovisual stimulation (who give quicker and/or more accurate responses for audiovisual stimuli when compared to unisensory stimuli) exhibit superadditive responses in the superior temporal sulcus, while those who do not show such a benefit show suppressive responses (Werner and Noppeney, 2010).

Superadditive responses are not the only type of response profile found during audiovisual integration. Previous studies have shown the coexistence of superadditive and subadditive neuronal populations in multisensory regions (e.g., Beauchamp, 2005; Beauchamp et al., 2004a;
Beauchamp et al., 2004b; Laurienti, Perrault et al., 2005; Stevenson et al., 2007). Informativeness of the auditory and visual modalities seems to be one of the consistent determinants of whether subadditivity or superadditivity is observed. For instance, multisensory interactions seem to be primarily subadditive for intact stimuli but additive for degraded stimuli (Werner and Noppeney, 2010). Nevertheless, the use of different criteria and contrasts/models might also impact the response profile found (for a discussion, see James and Stevenson, 2012). In the present study, in addition to measures of unimodal and bimodal processing and analyses of auditory and visual processing during audiovisual stimulation we also used superadditivity as a measure of audiovisual processing. For the superadditivity analyses, we made use of a conservative criterion where AudioVisual (AV) activity should be greater than the sum of the halves of Auditory (A) and Visual (V) activity $(\mathrm{AV}>(\mathrm{A} / 2+\mathrm{V} / 2))$.

Behavioral evidence suggests that typical and dyslexic readers differ in audiovisual speech processing (e.g., Francisco et al., 2017; Hayes et al., 2003; Norrix et al., 2006; Ramirez and Mann, 2005). Nevertheless, it is unclear whether and how the neural correlates for audiovisual speech processing are different in dyslexia when compared to the typical population. The few existent fMRI studies on this topic are inconsistent, use different methodologies and usually report results from rather small samples. Pekkola et al. (2006) tested for differences between typical and dyslexic adult readers in their processing of phonetically matching and conflicting audiovisual vowels. Dyslexic readers exhibited stronger activation than typical readers in motor speech regions, the left inferior parietal lobule, and in the ventral visual cortex. This result was interpreted as reflecting dyslexic readers' greater use of articulatory and visual information during phonetic processing of audiovisual speech, possibly to compensate for their difficulties in auditory speech perception. However, there were no unisensory control conditions or behavioral data, which makes it difficult to interpret these results. An opposite pattern was found by Kast et al. (2011): When compared to typical readers, adult dyslexic readers presented hypoactivity in the superior temporal gyrus and sulcus during an audiovisual word and pseudoword lexical decision task. In a similar line of research focusing on the role of the superior temporal gyrus in audiovisual speech perception, Ye et al. (2017) reported that, contrary to what was found for the adult typical readers, adult dyslexic readers did not show greater superior temporal activations for audiovisual stimuli when compared to unisensory stimuli. No behavioral differences were found between the groups. Recently, in an EEG-fMRI study, Karipidis et al. (2017) simulated the process of early reading acquisition in prereading children at varying risk for dyslexia by training artificial grapheme-phoneme associations. Learning and audiovisual integration measures correlated with both BOLD and EEG measures, suggesting that the ability to learn grapheme-phoneme associations might account for the degree of audiovisual integration in a distributed brain network. However, no low-risk sample was included in Karipidis et al., which would have allowed for more meaningful conclusions.

In the present study, we tested for differences between typical and dyslexic readers in BOLD activity using fMRI during a 1-back task in auditory, visual, and audiovisual speech conditions. Behavioral differences between typical and dyslexic readers reported in the literature might reflect difficulties in the integration of audiovisual speech and/or in the unisensory processing of the auditory or the visual component of speech. Therefore, we tested for differences between typical and dyslexic readers in audiovisual speech processing and in unisensory (auditory and visual) speech processing. In auditory speech, differences between typical and dyslexic readers might be found in Heschl's gyrus (e.g., Calvert et al., 1999; Callan et al., 2003; Möttönen et al., 2004; Pekkola et al., 2005) and in the superior temporal gyrus (e.g., Beauchamp et al., 2004a, 2010; Calvert et al., 2000). For visual speech, the middle temporal and the fusiform gyri are plausible candidates for group differences (e.g., Callan et al., 2003; Calvert et al., 1999; Calvert and Campbell, 2003; Macaluso et al., 2004; Stevenson et al., 2010; Wyk 
et al., 2010). Lastly, in audiovisual speech, differences between typical and dyslexic readers could be found in the superior temporal gyrus and in the supramarginal gyrus (Beauchamp et al., 2004a, 2010; Skipper et al., 2005; Stevenson et al., 2010, 2011), which are known to integrate audiovisual information, but also in any of the unisensory regions mentioned above. Given that superadditivity is argued to reflect audiovisual integration and benefit, we tested for differences between the groups in superadditivity, that is, whether activity is higher during audiovisual speech processing compared to mean activation level of unimodal auditory and visual speech processing.

Since reading is the core difficulty in dyslexia, we also tested the groups in two written stimuli conditions. In one condition, we tested natural reading of words. In another condition, we assessed more basic aspects of orthographic processing by asking participants to read illegal letter strings. The inclusion of these written stimuli allowed us to characterize the groups' neural response to graphemes. Additionally, given that grapheme-phoneme correspondences and audiovisual speech processing partly share neural circuitry (Blomert, 2011), neural responses to the written stimuli might contribute to the understanding of the responses to audiovisual speech material.

Confirmatory region-of-interest (ROI) analyses and exploratory whole-brain analyses were performed for both written stimuli and speech perception conditions. These two types of analyses have been shown to be complementary (Szycik et al., 2009).

\section{Materials and methods}

\subsection{Participants}

Forty-seven native Dutch undergraduate students were recruited. All participants had normal or corrected-to-normal vision and passed an audiometric hearing test (below $30 \mathrm{~dB}$ for 125, 250, 500, $1 \mathrm{~K}, 2 \mathrm{~K}, 3 \mathrm{~K}$, and $4 \mathrm{~K} \mathrm{~Hz}$ in both ears). All participants gave written informed consent prior to the experiment in accordance with the local ethics committee CMO region Arnhem-Nijmegen, the Netherlands. The participants received monetary compensation or course credits for their participation.

Participants' initial inclusion in the typical readers or dyslexic readers group depended on whether or not they had a prior diagnosis of dyslexia (one participant had been diagnosed during adulthood, all others received the diagnosis during childhood). In the Netherlands, a standardized protocol is followed for the diagnosis of dyslexia (Blomert, 2006). Dyslexia is diagnosed when a person scores at least 1.5 SD below their age norm on word and pseudoword reading measures and on two out of six tests assessing phonological awareness, letter knowledge, and rapid naming.

Participants' definitive inclusion was based on their performance in a reading task (see Section 2). Participants had to perform above the 30 th percentile on both the number of errors they made and the time they took to complete the reading task to be included in the typical readers group, or below the 30th percentile on either of these measures (reading errors and reading time) to be included in the dyslexic readers group. Five typical readers with no prior diagnosis of dyslexia who did not meet these criteria were excluded. Data from an additional typical reader could not be used due to malfunctioning of the scanner. The final sample therefore included 20 typical readers (8 men; age: $M=25.75$, $S D=4.06$ years) and 21 dyslexic readers (4 men; age: $M=24.89, S D$ $=2.70$ years). The median for typical readers was the 85 th percentile (range $=32$ nd to 96 th percentile) in reading errors and the 58th percentile (range $=30$ th to 98 th percentile) in reading time. The median for dyslexic readers was the 19 th percentile (range $=1$ st to 55th percentile) in reading errors and the 4 th percentile (range $=1$ st to 40th percentile) in reading time. Despite showing clear reading difficulties, all dyslexic readers were attending higher education. They had thus been successful in developing compensatory mechanisms.

\subsection{Materials and tasks}

2.2.1. Screening reading task (behavioral; performed outside the scanner)

Reading ability was assessed with a text-reading task from a standardized Dutch reading and writing battery for dyslexia diagnosis in adolescents and adults (Gl\&schr - Test voor gevorderd Lezen en Schrijven; De Pessemier and Andries, 2009). A 582-word text was presented to the participants, consisting of three paragraphs that ranged from easy to difficult. Participants were asked to read the text out loud (while being audio-recorded) as clearly and accurately as possible. Fast reading was discouraged. Silent pre-reading of the text was not allowed. When more than five seconds were taken to read a word, the experimenter would read the problematic word out loud and ask the participant to continue by reading the next word. Both reading time and reading errors were measured. Reading time was the total time in seconds taken to read the complete text. Reading errors were the total number of errors (omissions, additions, replacements, and inversions) made by the participants. Norms from the manual were used to transform raw scores for reading errors and reading time into percentiles.

\subsection{2. $f M R I$ written stimuli and speech perception tasks}

While lying in the scanner, participants performed a 1-back task, where they had to identify one repeated stimulus per block by pressing a button. The 1-back task was used to confirm that participants were attending to the stimuli, and was chosen for its simplicity. Additionally, its use allowed us to have the same task across conditions, diminishing taskdriven (perceptual and motor processing) variability. Five types of materials were used: printed words (written stimuli condition), illegal letter strings (written stimuli condition), and auditory, visual, and audiovisual consonant-vowel speech syllables (speech perception conditions). A sixth, speech-production condition also used printed words, but it will be reported elsewhere and therefore will not be discussed further.

The stimuli from both written stimuli conditions and the speech perception conditions are shown in Appendix A. In the printed words condition, fifteen two-syllable and fifteen three-syllable familiar words were presented one at a time on the screen. The stimuli were taken from a Dutch standardized reading test (Een-minuut-test, Brus and Voeten, 1999). In the illegal letter strings condition, consonant strings formed by five letters (e.g., $q f n t p$ ) were presented one at a time on the screen. These strings were formed by pseudo-randomly assigning consonants to five-letter strings which were orthographically and phonologically illegal and unpronounceable in Dutch. In the speech perception conditions, Dutch legal consonant-vowel (CV) syllables were presented auditory-only, visually-only, and audiovisually (that is, a video was shown in the audiovisual condition, its audio was muted in the visual condition and its video was hidden in the auditory condition). The videos of the visual and audiovisual conditions showed a female face and lasted for one second. To create these videos, a female native Dutch speaker was video-recorded pronouncing each CV syllable in isolation with a Sony Handycam DCR-SR190E. Audio was recorded at $44.1 \mathrm{kHz}$. Videos were digitized as uncompressed $400 \times 320$.avi files in PAL format and cut in Adobe Premiere Elements 11.0 (Adobe Systems, Mountain View, CA). The sound was presented at a comfortable listening level (clearly audible above the scanner noise) through in-ear MR compatible ear buds (Sensimetrics S14, http://www.sens.com/products/model-s14/).

Stimuli were presented blocked by condition. Each block consisted of seven stimuli presentations, where one out of six stimuli was presented twice. Five blocks were presented in each of the six conditions, amounting to a total of 30 blocks. Before each block, the upcoming condition was announced on a screen for $2500 \mathrm{~ms}$. Except in the speech production condition, each stimulus appeared on the screen for $1500 \mathrm{~ms}$, followed by an inter-trial interval of $500 \mathrm{~ms}$. Each block lasted approximately $14 \mathrm{~s}$. Participants were instructed to press a button if a stimulus was repeated. No speeded response was required, but the participants had to respond before the next stimulus was presented for 

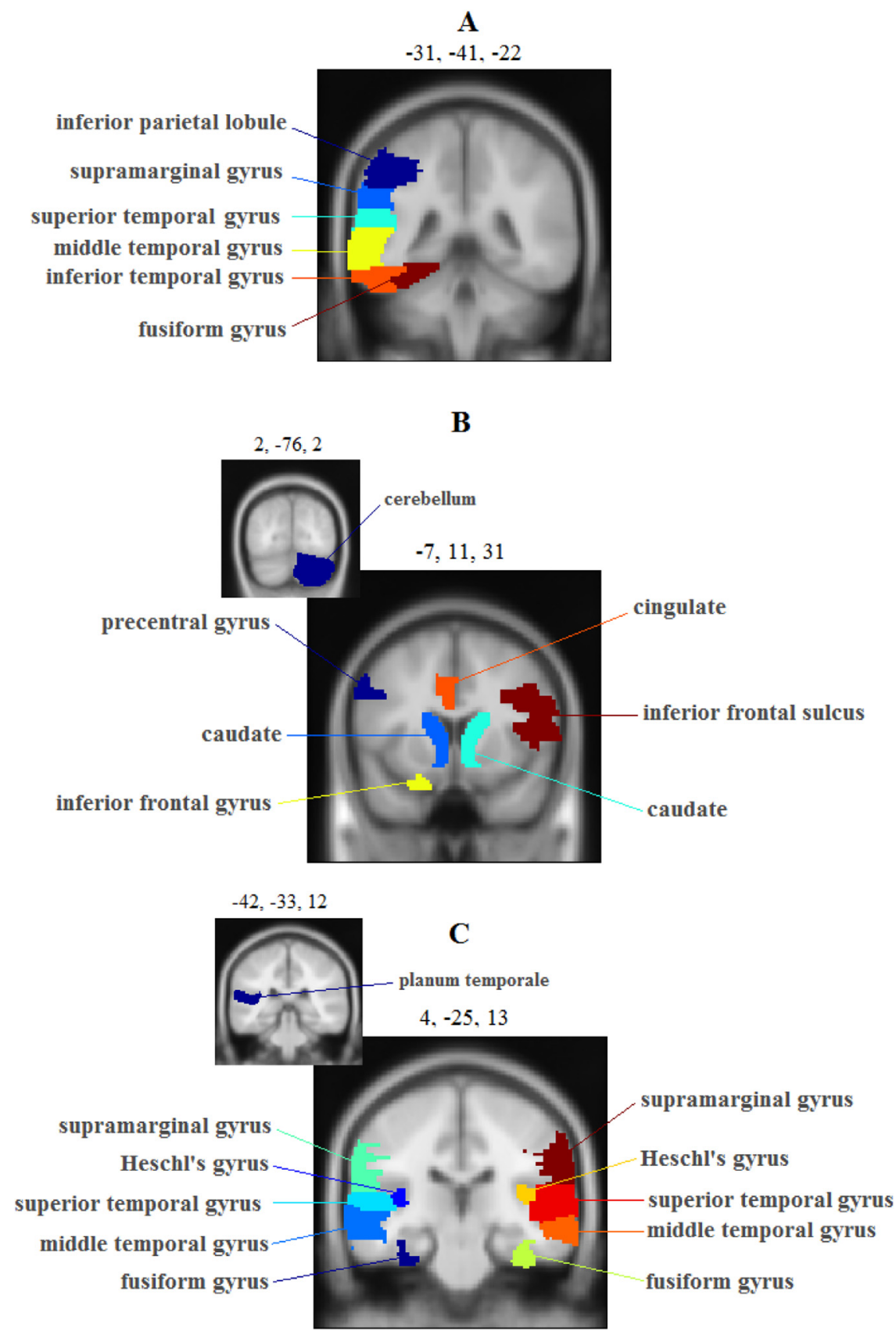

Fig. 1. ROIs extracted for the written stimuli conditions (A: areas previously found to be underactivated in dyslexia; B: areas previously found to be overactivated in dyslexia) and for the speech conditions (C).

the response to be registered. Reaction time was measured from the onset of the repeated stimulus. The order of the conditions was pseudorandomized for every participant, such that no consecutive blocks were of the same condition.

\subsection{Procedure}

The study was organized in two sessions, each lasting approximately one hour. In the first session, the reading assessment and the hearing screening were administered, together with other experimental tasks to be reported elsewhere. The scanning occurred in the second session. The task was explained outside of the scanner to ensure participants understood the instructions. Once in the scanner, participants were reminded of what was asked of them.

\subsection{MRI data acquisition}

The scans were performed using a Siemens 3T MAGNETOM Trio Tim MR system (Siemens Healthcare, Erlangen, Germany) with a 32channel head coil. A T1-weighted rapid acquisition gradient echo (MPRAGE) sequence was used to acquire an anatomical image of the whole brain: repetition time (TR): $2400 \mathrm{~ms}$; echo time (TE): $2.13 \mathrm{~ms}$; flip angle (FA): $8^{\circ}$; matrix size (MS): $384 \times 256$; field of view (FOV): $256 \mathrm{~mm}$, voxel size (VS); $1.0 \times 1.0 \times 1.0 \mathrm{~mm}^{3}$. Whole brain functional images were collected using a multi-band (accelerator factor of 8) T2*weighted sequence: TR: $735 \mathrm{~ms}$; TE: $39 \mathrm{~ms}$; FA: $52^{\circ}$; MS: $704 \times 704$; FOV: $210 \mathrm{~mm}$; VS: $2.4 \times 2.4 \times 2.4 \mathrm{~mm}$. Diffusion Tensor Imaging (DTI) and resting state MRI data were also acquired during the session, but will be reported elsewhere. 

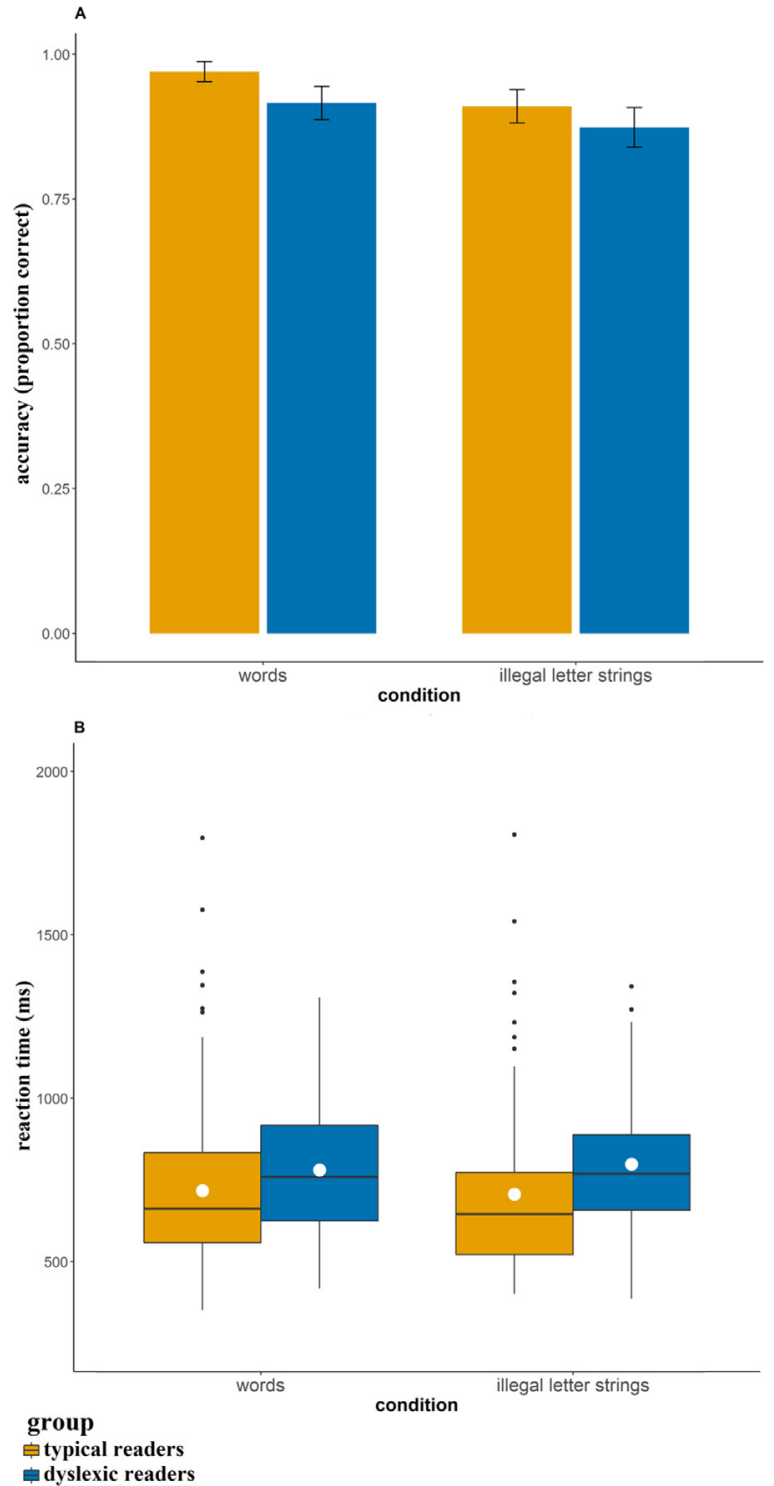

Fig. 2. Behavioral results for the written stimuli conditions (words, illegal letter strings) performed in the scanner by typical (orange) and dyslexic (blue) readers. A) Bar plots showing accuracy (proportion correct). B) Boxplots showing reaction time (in milliseconds). White dots indicate the condition mean. (For interpretation of the references to colour in this figure legend, the reader is referred to the web version of this article.).

\section{5. fMRI analyses}

\subsubsection{Pre-processing}

Image pre-processing and statistical analyses were performed with SPM8 (Statistical Parametric Mapping; Wellcome Department of Cognitive Neurology, London, UK; www.fil.ion.ucl.ac.uk) implemented in Matlab R2013a (MathWorks, Natick, MA). The first five volumes of each participant's functional EPI-data were excluded to allow for T1 equilibration. The first volume was used to realign the EPI images. The subject mean EPI was co-registered with the corresponding structural MRI using mutual information optimization. Both functional and structural scans were spatially normalized and transformed into a common Montreal Neurological Institute (MNI) space (resampled at voxel size $2 \times 2 \times 2 \mathrm{~mm}^{3}$ ), as defined by the SPM8 T1.nii template. Additionally, they were spatially filtered by convolving the functional images with an isotropic 3D Gaussian kernel $(8 \mathrm{~mm}$ full width at halfmaximum, FWHM).

\subsubsection{Confirmatory region-of-interest analyses}

To perform the region-of-interest (ROI) analyses, we first defined anatomical regions of interest using the WFU PickAtlas toolbox (AAL atlas) (Maldjian et al., 2004, 2003; Tzourio-Mazoyer et al., 2002) in SPM8 and the Harvard-Oxford probabilistic anatomical atlas (Makris et al., 2006) provided with the FMRIB Software Library (Smith et al., 2004; Woolrich et al., 2009). The planum temporale is not defined in the AAL atlas so, to define it, the Harvard-Oxford atlas was used. The MarsBaR toolbox (Brett et al., 2002) implemented in SPM8 was then used to extract the beta values for each of the ROIs, per group and in each of the conditions. Mean beta values for each of the ROIs were computed for every participant, and compared between groups for all conditions of interest. Bonferroni corrections were applied to correct for multiple comparisons.

For the written stimuli conditions, we extracted areas in which dyslexic readers have previously been shown to differ from typical readers in shallow orthographies, based on the meta-analysis performed by Martin et al. (2016). Those areas were divided into one large left occipitotemporoparietal cluster in which dyslexic readers showed underactivation: the left supramarginal gyrus, left superior temporal sulcus, left middle temporal gyrus, left fusiform gyrus, left inferior temporal gyrus, left inferior parietal lobule (Fig. 1A); and in regions in which dyslexic readers showed overactivation: the left precentral gyrus, left cingulate, left inferior frontal gyrus - pars orbitalis, bilateral caudate, right inferior frontal sulcus, and right cerebellum (Fig. 1B). For the speech perception conditions, we chose areas well described in the literature as responding to unimodal auditory material: superior temporal gyrus/sulcus and Heschl's gyrus (e.g., Calvert et al., 1999; Callan et al., 2003; Möttönen et al., 2004; Pekkola et al., 2005); to unimodal visual material (more specifically, to movement and faces): the middle temporal gyrus (e.g., Callan et al., 2003; Calvert et al., 1999; Calvert and Campbell, 2003) and fusiform gyrus (e.g., Calvert and Campbell, 2003; Macaluso et al., 2004; Stevenson et al., 2010; Wyk et al., 2010); and to audiovisual material: the superior temporal gyrus/sulcus and supramarginal gyrus (e.g., Beauchamp et al., 2004a, 2010; Calvert et al., 2000; Skipper et al., 2005; Stevenson et al., 2010, 2011). We additionally included the planum temporale as an area of interest, given previous literature on reduced responsiveness of that area to speech in dyslexia (e.g., Monzalvo et al., 2012). Fig. 1C depicts the speech perception ROIs.

\subsubsection{Exploratory whole-brain analyses}

The functional run was modeled using the General Linear Model (GLM) approach implemented in SPM8 for each participant. The regressors included the six conditions of interest (written stimuli, speech perception, and speech production conditions). These explanatory variables were temporally convolved with the canonical Hemodynamic Response Function (HRF) provided by SPM8. Each event was timelocked to the onset of the block with a duration of $16 \mathrm{~s}$. The design matrix included six head motion regressors (three translations, three rotations). A high-pass filter was implemented using a cut-off period of $128 \mathrm{~s}$ to remove low-frequency effects from the time series. For statistical analysis, contrast parameter images for each condition of interest were generated for each participant and then subjected to a secondlevel analysis (Penny et al., 2003), treating subjects as a random variable. For within group comparison for different conditions we used oneway ANOVAs. To test for differences between typical and dyslexic readers in each of the conditions we used 2-sample $t$-tests. All analyses included the offline reading measures (errors and time) as covariates, as a way of controlling for within-group differences. To detect the activation patterns, we applied cluster-size statistics using initial voxel level threshold at uncorrected $p<.001$ and family-wise corrected clustersize $P_{F W E}<0.05$ (Hayasaka and Nichols, 2003). To observe whether there was a superadditivity effect in the audiovisual condition relative to the auditory and the visual condition, we first computed a contrast 


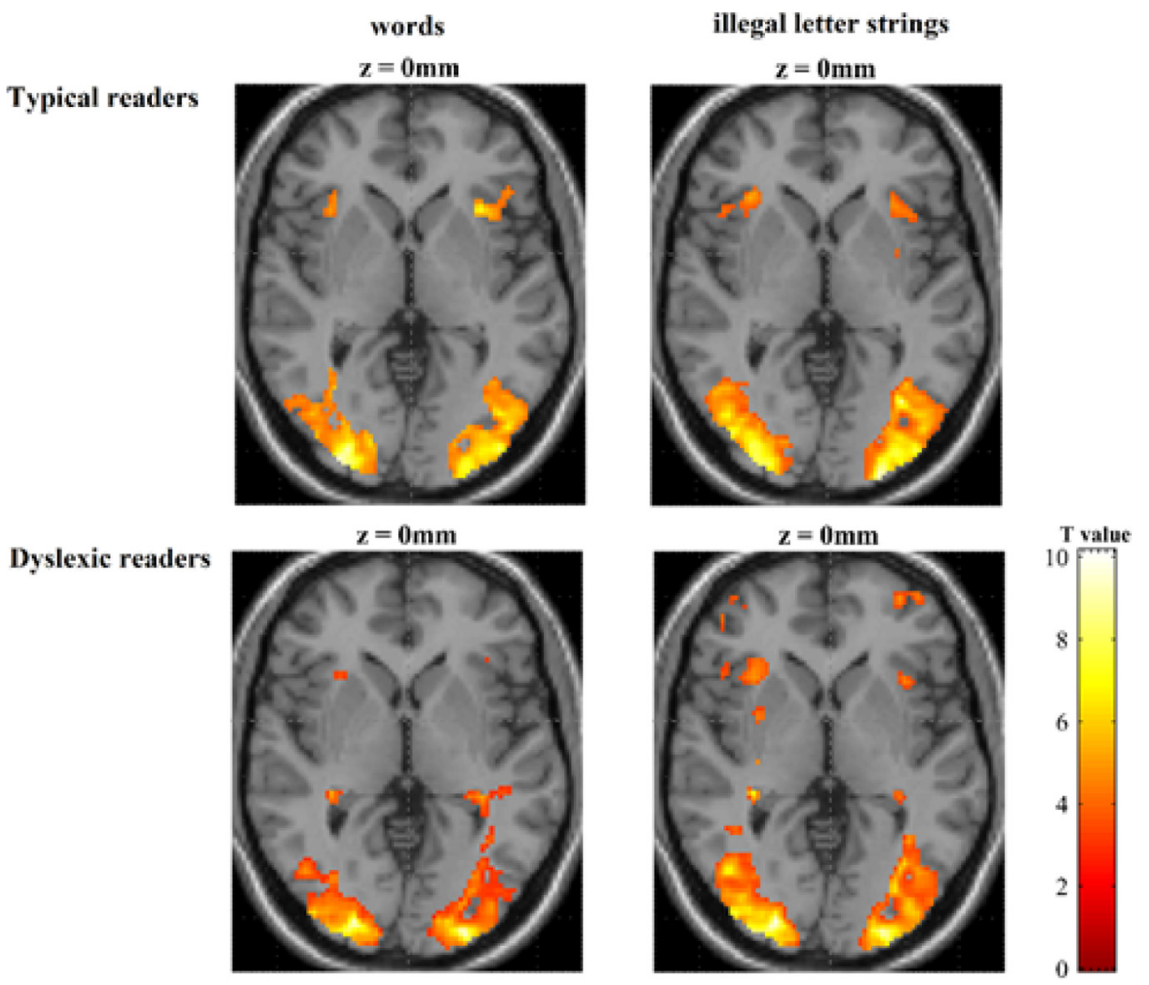

Fig. 3. Brain regions showing activity during each of the written stimuli conditions, per group, against baseline.

image weighting: 2 (audiovisual), -1 (auditory), -1 (visual) on the single subject level. This contrast image was tested using a one-sample $t$-test for the within-group effect, and a 2-sample $t$-test to compare between groups. This contrast assumes a linear relationship between audiovisual, visual and auditory conditions, in which significant voxels are those whose activation level in the audiovisual condition multiplied by two is greater than the summed activation level in the auditory and visual conditions. It thus effectively asks if, in activity, AV > (A/ $2+V / 2$ ). Depending on the region, however, the contribution of auditory and visual processing in the audiovisual condition may not be equal. Thus we also took a different (exploratory) approach where we looked into the audiovisual activity patterns in audition- or visionspecific brain areas by using functional masks to include only those voxels that were activated during unisensory inputs.

\section{Results}

\subsection{Screening reading task}

Two-sample independent means $t$-tests were used to test for group differences in the reading task. Welch corrections were applied to adjust the degrees of freedom where the assumption of the homogeneity of variances was violated. As expected, dyslexic readers made significantly more reading errors than typical readers (typical readers: $M=6.15, S D$ $=3.30$, dyslexic readers: $M=21.79, S D=14.17 ; t(19.85)=-4.69$, $p<.001, d=1.52$ ). Dyslexic readers also took significantly longer to read the text (typical readers: $M=244.10 \mathrm{~s}, S D=12.00$, dyslexic readers: $M=316.84, S D=37.02 ; t(21.57)=-8.17, p<.001, d$ $=2.64)$.

\section{2. fMRI task}

\subsubsection{Written stimuli conditions}

3.2.1.1. Behavioral results. Two dyslexic readers responded only to about $20 \%$ of the repeated trials and were therefore excluded from all further analyses. The false alarm rate in the written stimuli conditions was approximately $1 \%(M=0.01, S D=0.03)$. Fig. 2 shows the behavioral performance (reaction time and accuracy) in each of the conditions (words and illegal letter strings), per group.

Mixed-effect models were implemented to analyze the data, using the lmer function in the lme4 package (Bates et al., 2014) in R (version 3.1.2, R Core Team, 2014). Two separate models were built for reaction time and accuracy (numeric dependent variables). In both models, group (typical readers $=-0.5$, dyslexic readers $=0.5$ ) and condition (words $=-0.5$, illegal letter strings $=0.5$ ) were contrast-coded fixed factors. Subjects were added as a random factor, along with by-subject slope adjustments for condition (Barr et al., 2013). Models were fit using the maximum likelihood criterion. P-values were estimated using Satterthwaite approximations. For both accuracy and reaction times, there were no significant differences between groups or conditions or significant interactions between the two factors (all $p>.05$ ).

\subsubsection{Imaging results}

3.2.1.2.1. Activation patterns. Fig. 3 and Table B1 (in Appendix B) show the significantly activated clusters for each written stimuli condition (compared to baseline; baseline was at the inter-block interval, which consisted of a fixation cross on the screen for $16 \mathrm{~s}$ ), per reading group.

3.2.1.2.2. ROI analysis. As described in the fMRI analyses section, twelve anatomical ROIs were chosen for this analysis. Six of those ROIs were previously shown in studies to be underactivated in dyslexic readers, when compared to typical readers (left supramarginal gyrus (SMG), left superior temporal gyrus (STG), left middle temporal gyrus (MTG), left fusiform gyrus (FUSF), left inferior temporal gyrus (ITG), and left inferior parietal lobule (IPL)). The other six have been reported to be overactivated in dyslexic readers, when compared to typical readers (left precentral gyrus (PG), left cingulate (CIN), left inferior frontal gyrus - pars orbitalis (IFG), bilateral caudate (CAr/CAl), right inferior frontal sulcus (IFS), and right cerebellum (CB)). Fig. 4 shows mean activation per group and ROI in the words condition (panel A) and in the illegal letter strings condition (panel B). The overactivation/ underactivation pattern found in the present study is generally 


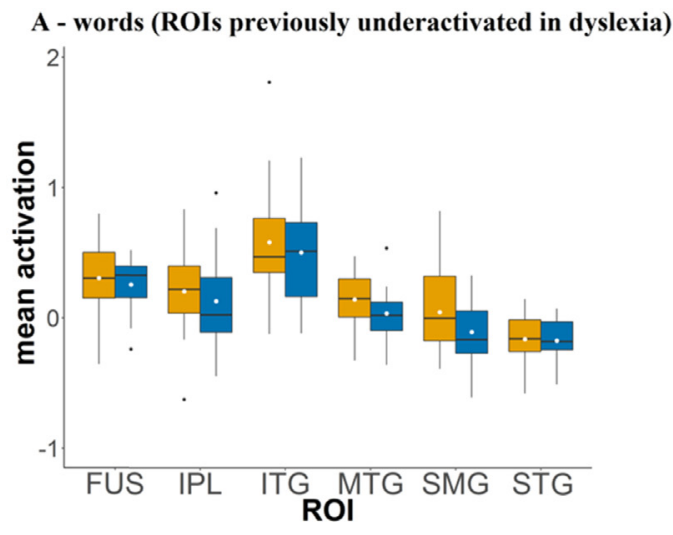

C - illegal strings (ROIs previously underactivated in dyslexia)

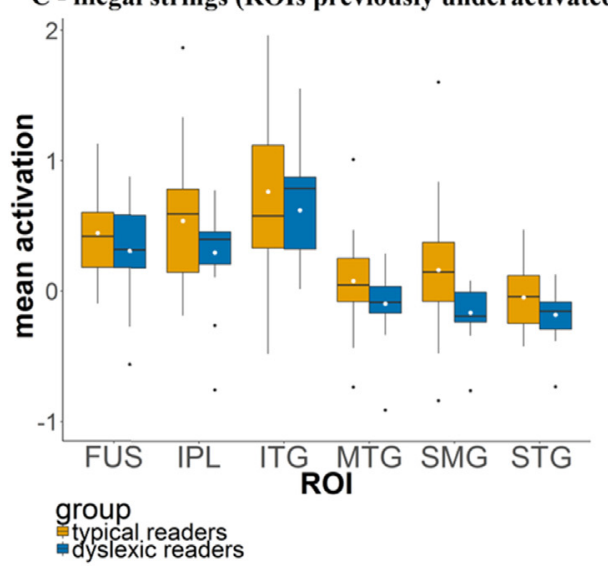

B - words (ROIs previously overactivated in dyslexia)

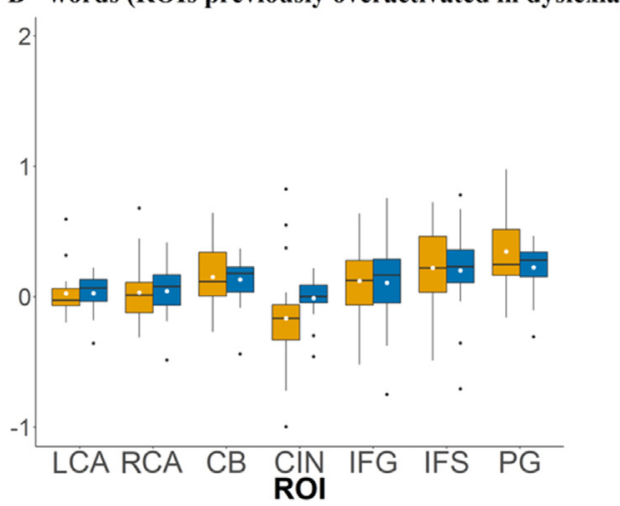

D - illegal strings (ROIs previously overactivated in dyslexia)

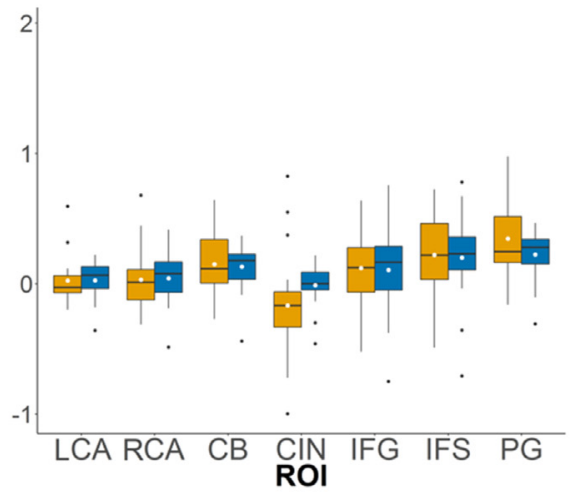

Fig. 4. Boxplots showing mean parameter estimates extracted from the voxels in the anatomically defined ROIs per group in the words condition (panel A: areas previously shown to be underactivated in dyslexic readers; panel B: areas previously shown to be overactivated in dyslexic readers) and in the illegal letter strings condition (panel C: areas previously shown to be underactivated in dyslexic readers; panel D: areas previously shown to be overactivated in dyslexic readers). White dots indicate the mean. SMG: left supramarginal gyrus; STG: left superior temporal gyrus; MTG: left middle temporal gyrus; FUSF: left fusiform gyrus; ITG: left inferior temporal gyrus; IPL: left inferior parietal lobule; PG: left precentral gyrus; CIN: left cingulate; IFG: left inferior frontal gyrus - pars orbitalis; RCA/LCA: bilateral caudate; IFS: right inferior frontal sulcus; CB: right cerebellum.

consistent with that reported previously (Martin et al., 2016), with the exception of the illegal letter strings condition where, in the areas previously reported to be overactivated in dyslexic readers, there was a tendency towards underactivity in some regions (cerebellum, inferior frontal gyrus, inferior frontal sulcus, and precentral gyrus).

To test for differences between the groups in these ROIs, general linear models (GLMs) were carried out on the mean of the extracted parameter estimates per condition (words and illegal letter strings) and per type of ROI (previously reported to be underactivated or overactivated in dyslexic readers). Activation was the dependent variable, while group and ROI were fixed factors.

In the words condition, there was neither an effect of group nor an interaction between group and ROI. ROI had a significant effect in both underactivated areas: $F(5,222)=28.11, p<.001\left(\eta^{2}=0.39\right)$ and overactivated areas: $F(6,259)=5.27, p<.001\left(\eta^{2}=0.11\right)$ showing that the level of activation differed among the ROIs.

In the illegal letter strings condition, there was neither an effect of group in the overactivated ROIs nor an interaction between group and ROIs. Group had an overall effect in the underactivated ROIs: $F$ $(1,222)=16.12, p<.001\left(\eta^{2}=0.07\right)$. Bonferroni-corrected post-hoc comparisons revealed only one significant effect: that typical readers showed increased activity in the left supramarginal gyrus when compared to the dyslexic readers: $t(37)=1.86, p<.05$. ROI had a significant effect in both the underactivated areas: $F(5,222)=26.01$, $p<.001\left(\eta^{2}=0.37\right)$ and the overactivated areas: $F(6,259)=8.43$, $p<.001\left(\eta^{2}=0.16\right)$.

3.2.1.2.3. Whole brain analysis. Differences between typical and dyslexic readers were also tested through an exploratory whole-brain analysis. We tested for differences in each of the conditions against baseline (inter-block interval, which consisted of a fixation cross on the screen for $16 \mathrm{~s}$ ), focusing on two different contrasts (typical readers $>$ dyslexic readers and dyslexic readers $>$ typical readers). In the illegal letter strings condition, typical readers showed increased activity in a left supramarginal gyrus cluster (cluster size: 137 voxels; local maximum $[x=-56, y=-24, z=40]$ ), when compared to dyslexic readers. No clusters with significant group differences were found in the word condition.

\subsubsection{Speech perception conditions}

3.2.2.1. Behavioral results. Fig. 5 shows the behavioral performance (reaction time and accuracy) per group in the auditory, the visual, and the audiovisual speech perception conditions. Across all speech perception conditions, the false alarm rate was $14 \%(S D=0.16)$.

Mixed-effect models were implemented as described for the written stimuli conditions. Group was contrasted-coded (typical readers = -0.5 , dyslexic readers $=0.5$ ). The auditory condition was mapped onto the intercept. In terms of accuracy, dyslexic readers detected fewer repetitions than typical readers $(\beta=-0.16$, SE $=0.08, p=.04)$. Although in general participants were all able to perform the task with relatively high accuracy, performance was, as expected, worse in the visual condition $(\beta=-0.15, \mathrm{SE}=0.05, p<.001)$. There were no significant interactions between group and any of the conditions. Concerning reaction times, overall, more time was taken in the visual condition $(\beta=59.86, \mathrm{SE}=27.31, p=.04)$. Furthermore, dyslexic 

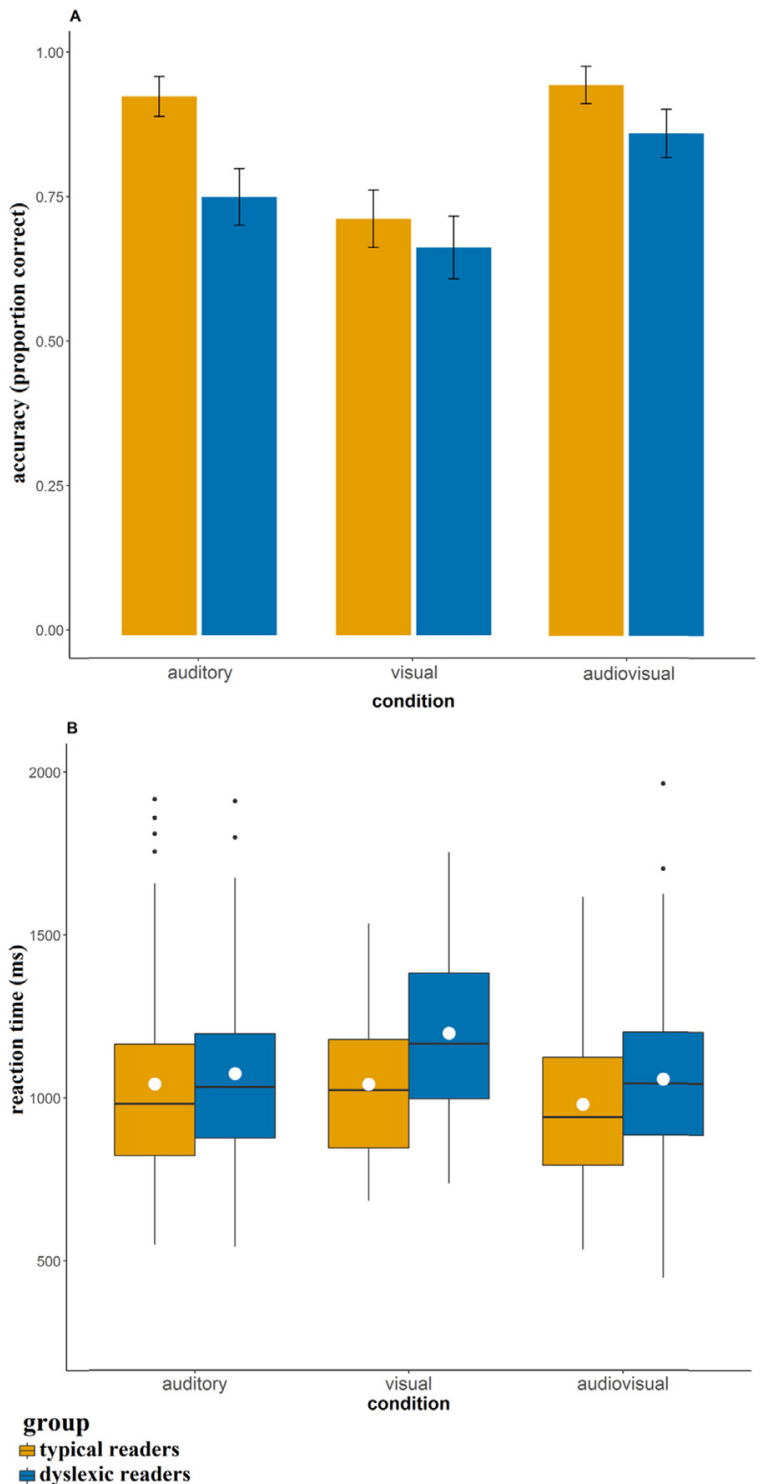

Fig. 5. Behavioral results for the speech perception conditions (auditory, visual, audiovisual) performed in the scanner by typical (orange) and dyslexic (blue) readers. A) Bar plots showing accuracy (proportion correct). B) Boxplots showing reaction time (in milliseconds). White dots indicate the mean. (For interpretation of the references to colour in this figure legend, the reader is referred to the web version of this article.).

readers were slower than the typical readers, but only significantly so in the visual condition $(\beta=114.11, \mathrm{SE}=54.63, p=.04)$. Additionally, we tested for differences in audiovisual benefit between typical and dyslexic readers. The audiovisual benefit was calculated by subtracting accuracy in the auditory condition from accuracy in the audiovisual condition and dividing that value by the accuracy in the auditory condition subtracted from 1: (AV-A)/(1-A). No differences were found between the groups $(p>.05)$, that is, at least behaviorally, both typical and dyslexic readers seem to have benefitted similarly from audiovisual stimulation.

\subsubsection{Imaging results}

3.2.2.2.1. Activation patterns. Fig. 6 and Table B2 (in Appendix B) show the significantly activated clusters for each condition (compared to baseline), per reading group.

3.2.2.2.2. ROI analysis. As described in the fMRI analyses section, different anatomical ROIs were chosen per condition for this analysis.
The auditory condition analysis included the superior temporal gyrus, Heschl's gyrus and the left planum temporale; the visual condition analysis included the fusiform gyrus, the middle temporal gyrus and the left planum temporale; the audiovisual condition analysis focused on the ROIs used in the unimodal conditions and, additionally, on the supramarginal gyrus. Fig. 7 shows the mean of the extracted parameter estimates of the voxels within the anatomically defined ROIs per condition and group.

GLMs were performed on the mean of the extracted parameter estimates for each of the conditions for the ROIs listed above, following the same procedure as in the analyses of the written stimuli conditions. Here, the models were built per condition. Activity level was the dependent variable. Group, ROI, and ROI laterality were fixed factors. In the auditory condition there was an effect of group: $F(1,185)=4.25$, $p<.05\left(\eta^{2}=0.02\right)$, with dyslexic readers showing decreased activity in the regions of interest (typical readers: $M=0.813, S D=0.034$; dyslexic readers: $M=0.710, S D=0.035)$. ROI also had a significant effect: $F(2,185)=20.13, p<.001\left(\eta^{2}=0.18\right)$. No significant effects of ROI laterality or of any of the interactions were found. In the visual condition, there was again a significant effect of group ( $F$ $(1,185)=3.94, p<.05\left(\eta^{2}=0.02\right)$; typical readers: $M=0.405$, $S D=0.028$; dyslexic readers: $M=0.320, S D=0.029)$ and of ROI $(F$ $\left.(2,185)=12.91, p<.001\left(\eta^{2}=0.12\right)\right)$. In the audiovisual condition, group had an overall significant effect: $F(1,407)=8.94, p<.01\left(\eta^{2}\right.$ $=0.02$ ), with dyslexic readers showing decreased activation compared to the typical readers (typical readers: $M=0.573, S D=0.023$; dyslexic readers: $M=0.474, S D=0.024)$. The effect of ROI was also significant: $F(5,407)=55.63, p<.001\left(\eta^{2}=0.41\right)$.

3.2.2.2.3. Superadditivity. Superadditivity was first tested with the contrast $\mathrm{AV}>(\mathrm{A} / 2+\mathrm{V} / 2)$ (Barraclough, Xiao, Baker, Oram, and Perret, 2005; Kronschnabel et al., 2014; Meredith and Stein, 1983, 1986). The same ROIs as used in the audiovisual condition were also used in the superadditivity analysis. Table B3 (in Appendix B) shows the significantly activated clusters for $\mathrm{AV}>\mathrm{A}+\mathrm{V}$, per reading group. Fig. 8 shows the mean parameter estimates extracted from the voxels in the anatomically defined ROIs for the superadditivity contrast.

The GLM approach described previously was also applied here. The difference score was the dependent variable. Group, ROI, and ROI laterality were fixed factors. There was a significant effect of group: $F$ $(1,407)=4.60, p<.05\left(\eta^{2}=0.011\right)$, with dyslexic readers showing smaller difference scores compared to the typical readers (typical readers: $M=0.571, S D=0.033$; dyslexic readers: $M=0.470, S D=$ 0.034). The effect of ROI was significant: $F(5,407)=32.12, p<.001$ $\left(\eta^{2}=0.29\right)$. The effect of ROI laterality and all interactions were not significant.

Group differences in audiovisual processing might result from the processing of the auditory or of the visual component during audiovisual stimulation. We carried out two additional exploratory analyses to test these possibilities: the first analysis looked at auditory processing during the presentation of audiovisual stimuli and the second analysis focused on visual processing during the presentation of audiovisual stimuli.

To test for differences between the groups in auditory-related processing during the audiovisual condition, we first ran two-sample $t$ tests, comparing typical and dyslexic readers in the areas where, during the audiovisual condition, audiovisual activity was greater than visual activity $(\mathrm{AV}>\mathrm{V})$. We assumed that this contrast would show areas that are involved in either auditory processing or in processing related to the integration of audiovisual information. Since we were exclusively interested in the regions showing brain activation specific to unimodal auditory processing, we then applied an inclusive mask selecting the areas in which activity was greater during the auditory condition than during the visual condition, per group (i.e., all the voxels that showed greater activation for auditory relative to visual condition applying voxel level threshold $p<.001$, uncorrected, for the contrast $\mathrm{A}>\mathrm{V}$ ). This analysis revealed no significant clusters, that is, there were no 

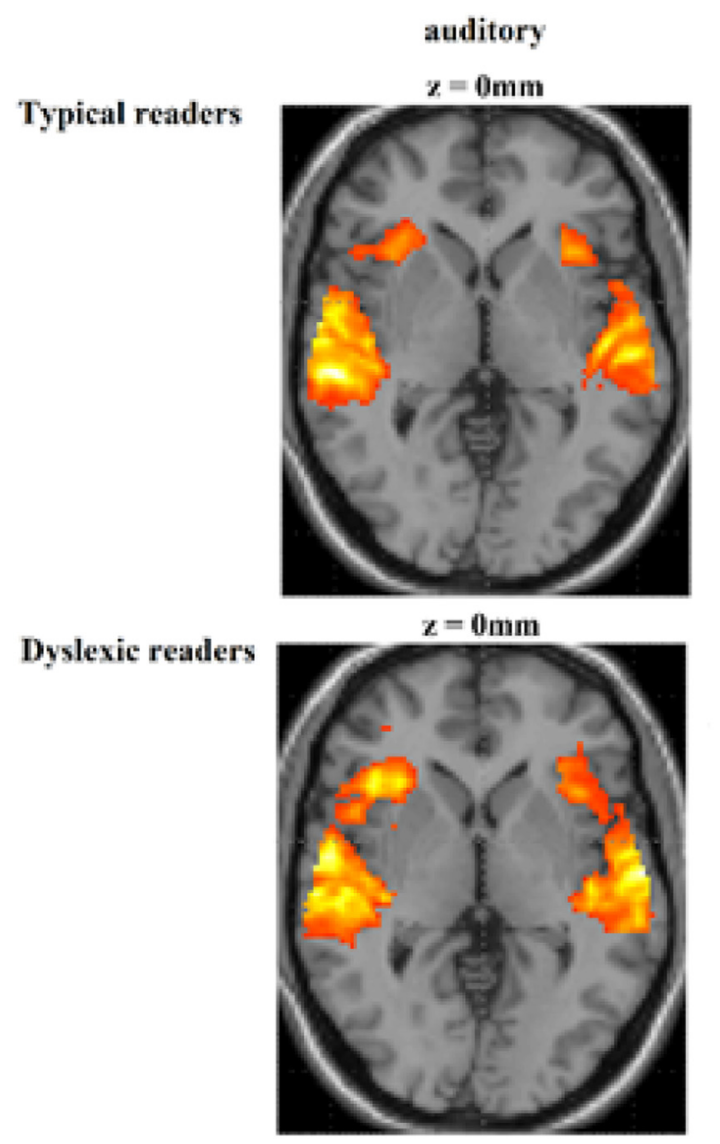
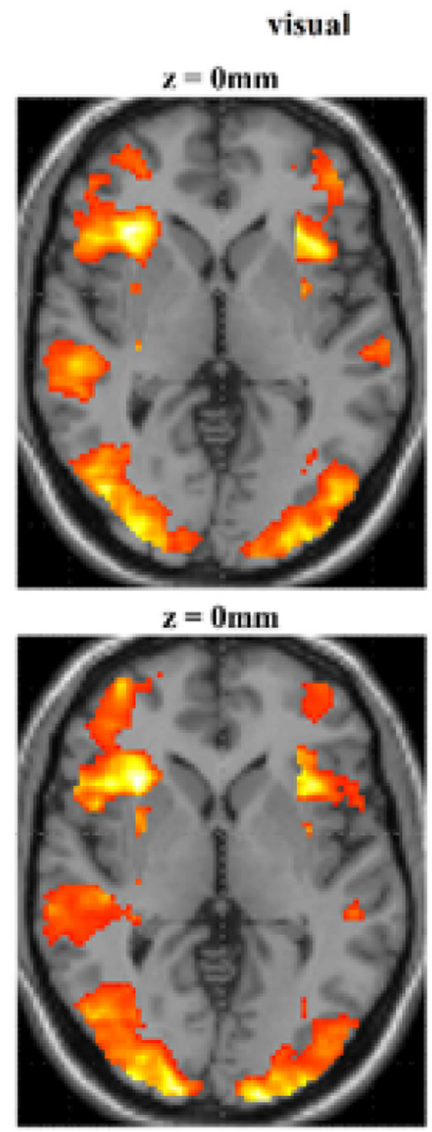
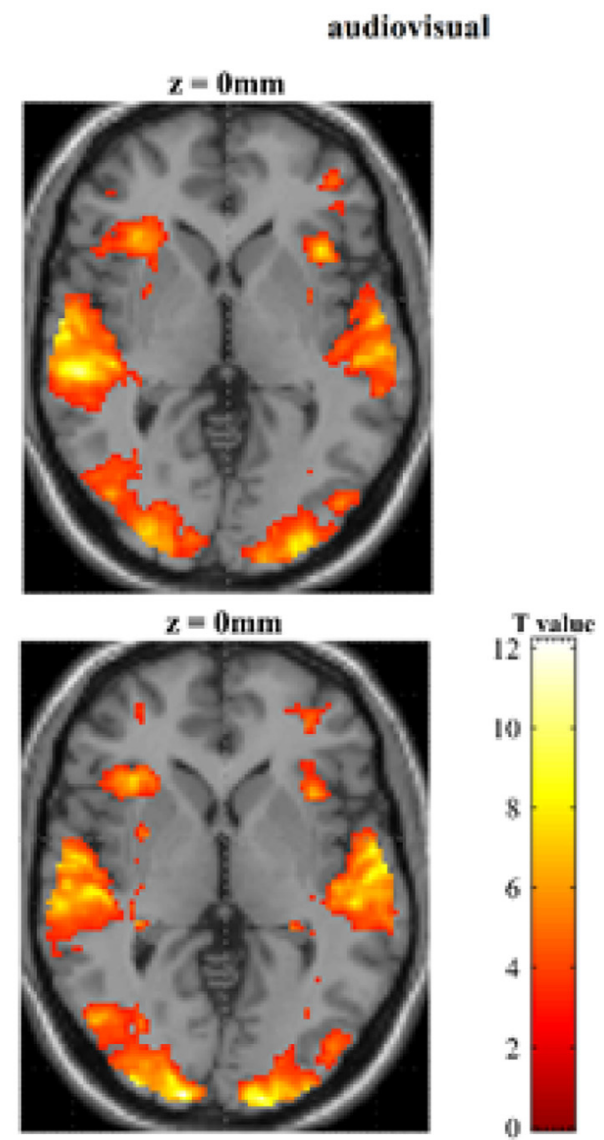

Fig. 6. Brain regions showing activity during each of the audiovisual speech conditions, per group, against baseline.

differences between typical and dyslexic readers in areas where $\mathrm{AV}>\mathrm{V}$ and $\mathrm{A}>\mathrm{V}$.

To test for differences between the groups in visually-related processing during the audiovisual condition, we compared the groups in the areas where audiovisual activity was greater than auditory activity $(\mathrm{AV}>\mathrm{A})$, specifically focusing on the areas that also showed brain activation specific to unimodal visual processing $(\mathrm{V}>\mathrm{A})$. This analysis revealed a significant cluster (voxel level threshold $p<.001$; significant at cluster level, $p=.01$, FWE-corrected) in the left fusiform gyrus (typical readers $>$ dyslexic readers; cluster size: 34 voxels; local maximum $[x=-30, y=-66, z=-12]$ (see Fig. 9).

In order to specify whether the left fusiform area reported above was more likely to reflect processing of faces or visual words, we contrasted activity in the condition with written words with that found in the visual speech condition and detected clusters in the anatomically defined fusiform gyrus (bilateral mask, created with the WFU PickAtlas toolbox, as previously described). The contrast written words $>$ visual speech (visual speech processing) revealed clusters around the coordinates $x=-29 \pm 1, y=-39 \pm 1, z=-14 \pm 2$ within the fusiform gyrus. The contrast visual speech $>$ written words (face processing) revealed clusters around $x=37 \pm 5, y=-63 \pm 13$, $z=-16 \pm 2$ and $x=-32 \pm 4, y=-67 \pm 7, z=-17 \pm 1$. The peak activity of the fusiform cluster identified in the group contrast (typical $>$ dyslexic) above (around the coordinates $x=-30$, $y=-66, z=-12$ ) was closer to the cluster showing greater activation for the visual speech condition than to the cluster showing greater activation for the words condition. Thus, the visual processing benefit in the typical readers during the audiovisual condition could be due to additional processing of faces during the audiovisual condition. This possibility was further confirmed by additional analyses (see Fig. 10), which demonstrated that the specific fusiform cluster showed increased activity during the visual condition (when compared to the auditory condition, $p<.001$ ). Importantly, these analyses also revealed that, compared to typical readers, dyslexic readers showed decreased activation in the fusiform cluster during the visual $(p<.05)$ and the audiovisual $(p<.05)$ conditions.

3.2.2.2.4. Whole brain analysis. Differences between typical and dyslexic readers were also tested through an exploratory whole-brain analysis. We tested for differences between groups (typical readers $>$ dyslexic readers and dyslexic readers $>$ typical readers) in each of the conditions (against baseline). In the auditory condition, a cluster in the right precuneus (cluster size: 95 voxels; local maximum $[x=10, y=-50, z=28]$ ), revealed more activity for the dyslexic than for the typical readers. No clusters with significant group differences were found for the reverse contrast for the auditory condition, or in either contrast direction for the visual and audiovisual conditions.

Since there was overlap in the variance in reading performance across the typical and dyslexic readers, we also ran an exploratory correlational analysis across all participants, independent of whether they had a diagnosis of dyslexia. We specifically tested correlations between activation levels and reading performance. After correcting for multiple comparisons, none of the areas showed a correlation with this index in any of the conditions. Additionally, we performed regression analyses at the whole brain level adding reading errors and reading time as predictors. Apart from activity increase that correlated with the number of errors in the left middle frontal gyrus for auditory and visual conditions and a cluster in the right superior frontal gyrus of the auditory condition, none of the areas showed correlation with the reading measures for any of the conditions in the areas of interest. 

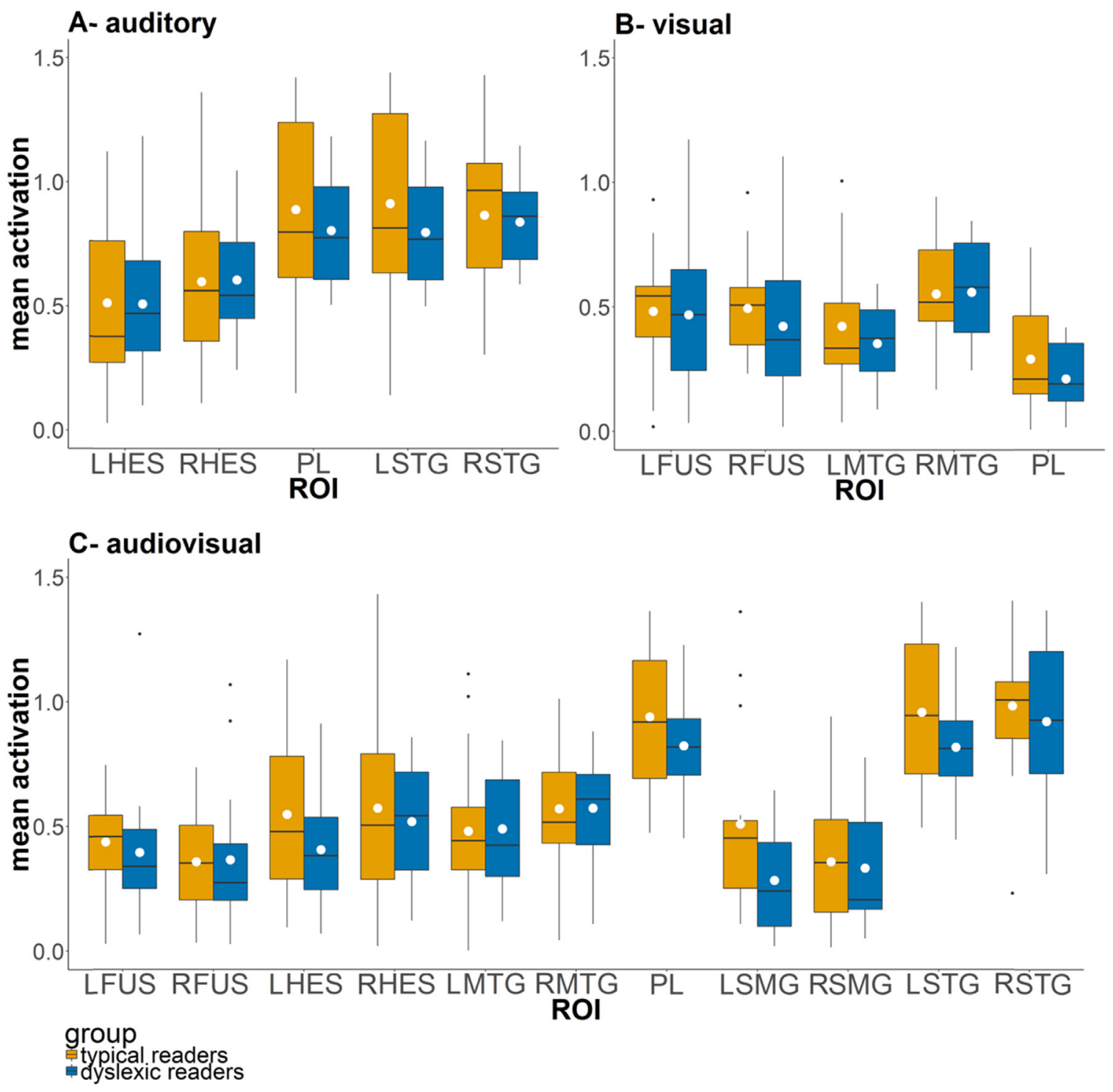

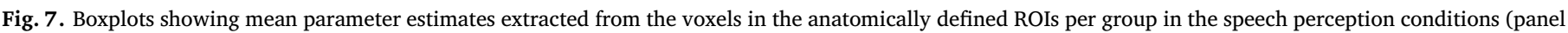
A: auditory, panel B: visual, panel C: audiovisual). White dots indicate means. STG: superior temporal gyrus; HES: Heschl's gyrus; MTG: middle temporal gyrus; FUS: fusiform gyrus; SMG: supramarginal gyrus; PL: left planum temporale; R: right; L: left.

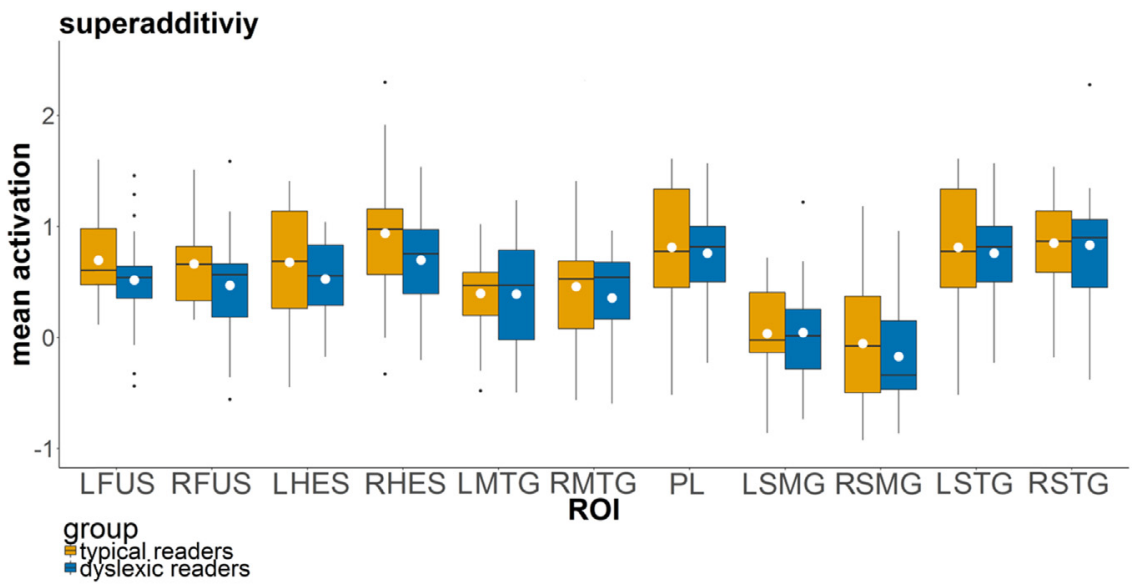

Fig. 8. Boxplots showing mean parameter estimates extracted from the voxels in the anatomically defined ROIs for the superadditivity contrast. White dots indicate mean. Positive numbers reflect $\mathrm{AV}>\mathrm{A}+\mathrm{V}$. STG: superior temporal gyrus; HES: Heschl's gyrus; MTG: middle temporal gyrus; FUS: fusiform gyrus; SMG: supramarginal gyrus; PL: planum temporale; R: right; L: left. 

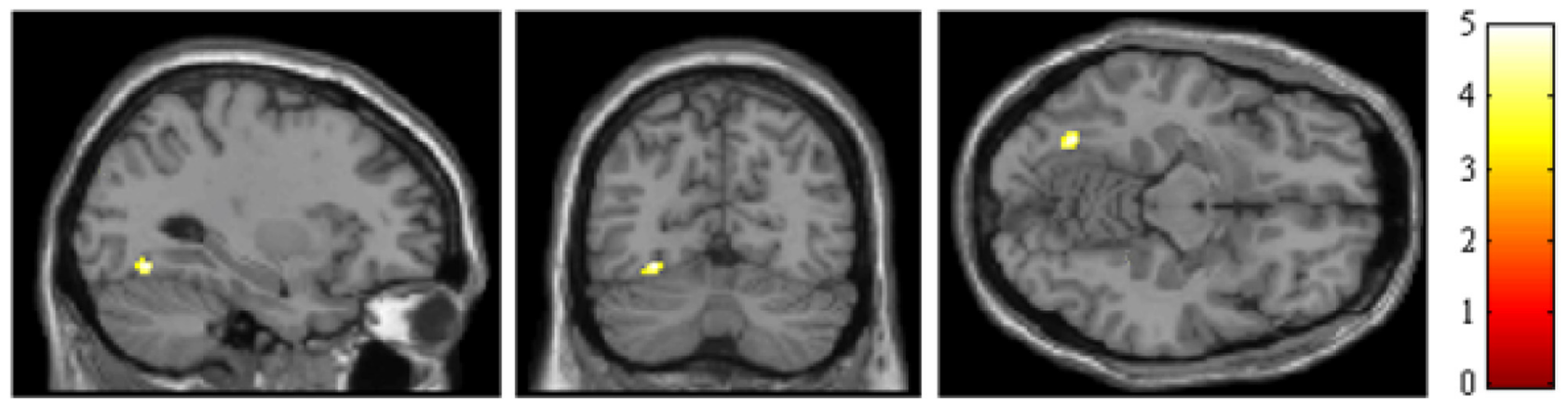

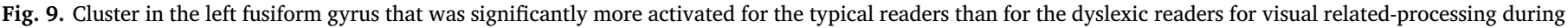
the audiovisual condition.

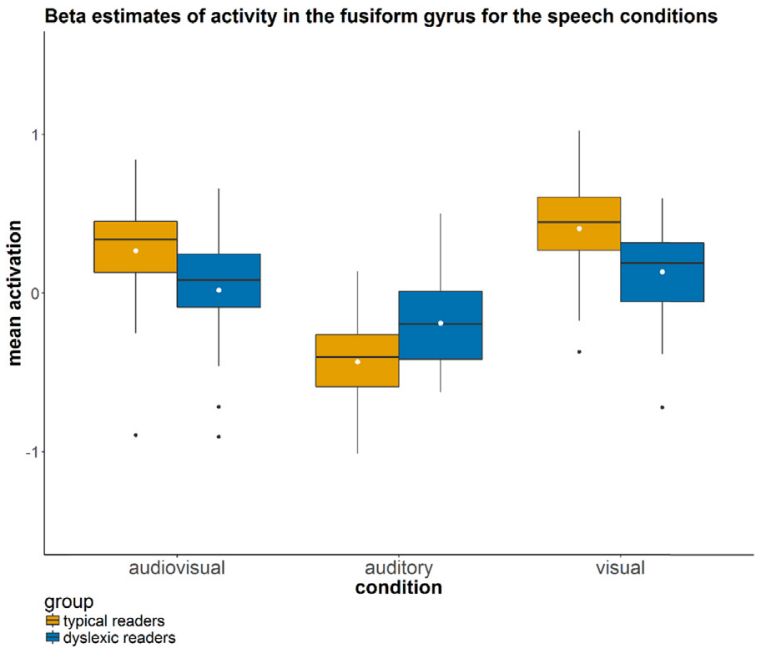

Fig. 10. Boxplots showing mean parameter estimates extracted from the voxels in the fusiform gyrus (Fig. 8) per speech condition and by group. White dots indicate mean.

\section{Discussion}

The present study examined the neural correlates of audiovisual speech processing in groups of adult dyslexic and typical readers. Differences between the groups could indicate the presence of a general audiovisual deficit in dyslexia, rather than a deficit limited to the inefficient processing of (audiovisual) associations between letters and speech sounds. We collected behavioral and imaging data from typical and dyslexic adult readers who were tested on written stimuli processing and speech perception while performing a 1-back task. Additionally, we tested for differences in superadditivity between typical and dyslexic readers. Behaviorally, when compared to typical readers, dyslexic readers were slower and less accurate in the 1-back task, particularly in the visual speech condition. When performing the 1-back task on illegal letter strings, dyslexic readers showed reduced activation in the supramarginal gyrus. In the speech perception conditions, dyslexic readers showed less neural activation during the auditory, the visual, and the audiovisual conditions. This result suggests that (audiovisual) speech processing differs between dyslexic and normal readers and that this difference is a general one (i.e., it is not limited to print-sound associations). Furthermore, when compared to typical readers, dyslexic readers presented decreased activation in a cluster in the fusiform gyrus. Less activation in the fusiform gyrus while processing audiovisual speech in dyslexic readers indicates reduced processing of facial information during audiovisual speech perception. Since differences were found not only in the audiovisual speech condition, but also for auditory and visual processing, one possibility is that deficits in the processing of the unisensory stimuli create the differences in audiovisual processing.

In the two written stimuli conditions in the scanner, there were no significant behavioral differences in the 1-back task between the groups. The neuroimaging results revealed no differences between typical and dyslexic readers in the word condition. The dyslexic readers tested in the present study were highly educated adults, who have most certainly developed compensatory strategies to deal with their reading difficulties. Taken together with the fact that we presented words with a high degree of familiarity, this might explain why no differences were found between the groups while the participants were detecting word repetitions. Additionally, the groups' overlap in reading performance could have contributed to the lack of differences observed here. In the illegal letter strings condition, however, dyslexic readers showed decreased activation in the supramarginal gyrus when compared to the typical readers. This finding might suggest that typical readers, to a greater extent than dyslexic readers, processed the strings phonologically, as the supramarginal gyrus has been suggested to play an important role in phonological processing and in the mapping of graphemes to phonemes (Caplan et al., 2009; Ojemann et al., 1989; Paulesu et al., 1996; Rumsey et al., 1997; Sandak et al., 2004). Grapheme-phoneme correspondences could happen as a task strategy (rehearsal of sequences), but also automatically (given that these strings were formed by letters). That is, participants could have sounded out the letter names of the consonant strings using grapheme-to-phoneme conversion in order to perform the 1-back task. Thus, consistent with previous studies (Blau et al., 2009; Kast et al., 2011; Kronschnabel et al., 2014), this result suggests deficient grapheme-phoneme processing, even in highly educated dyslexic adults. That this difference between the groups only emerged in the illegal letter strings condition might be due to the increased difficulty (in terms of memory load) of this condition when compared to the words condition. Given that working memory has been consistently associated with reading (e.g., Christopher et al., 2012; Swanson et al., 2006; Swanson et al., 2009), an alternative interpretation is that the unfamiliar illegal letter strings resulted in a higher working memory load, particularly for the dyslexic readers, which could have impacted the neural activation pattern of dyslexic readers. Finally, the group difference in neural activation might also reflect a difference in the use of verbal rehearsal as a strategy to complete the task in the illegal letter string condition.

When detecting repetitions in the 1-back task on speech materials, 
dyslexic readers were less accurate than the typical readers in all conditions and slower in the visual speech condition. These results fit with previous behavioral evidence of an audiovisual speech perception deficit in dyslexia (e.g., Francisco et al., 2017; Hayes et al., 2003; Norrix et al., 2006; Ramirez and Mann, 2005). The ROI analysis revealed differences between the groups in all speech perception conditions: Dyslexic readers showed decreased neural activation during the auditory, the visual, and the audiovisual conditions. This result suggests not only that there are general audiovisual speech processing differences between dyslexic and normal readers, but also that these differences might be, at least in part, explained by differences in the processing of both the auditory and the visual components of speech.

Diminished activity in dyslexia during auditory, visual, and audiovisual speech processing could reflect a functional disruption. Decreased neural activity during the auditory condition is consistent with studies showing differences between typical and dyslexic readers on several auditory tasks (see Rosen, 2003, for a review of the literature). Moreover, it is in accordance with past neuroimaging evidence of reduced BOLD responses in dyslexic readers for unisensory presentations of speech sounds (Blau et al., 2009). Functional neuroimaging studies on linguistic processing in dyslexia have mostly focused on the processing of written material, showing consistent decreased neural activation (e.g., Shaywitz et al., 1998, 2003). The finding that dyslexic readers show less activation than typical readers during visual speech processing is consistent with and extends such evidence. Furthermore, this finding is in accordance with the present behavioral results and with other studies showing behavioral difficulties with speechreading in dyslexic readers (de Gelder and Vroomen, 1998).

The diminished activation during audiovisual speech seen in dyslexic readers is, once again, consistent with the behavioral results and with the well-established inefficient processing of (audiovisual) letterspeech sound associations in dyslexia (Blau et al., 2009, 2010; Kast et al., 2011; Kronschnabel et al., 2014). Our finding adds to previous results, showing that the audiovisual deficit in dyslexia may not be specific to letter-speech sound associations, but might instead be broader in nature. One possibility is that a general audiovisual deficit impairs the adequate establishment of audiovisual associations, such as associations between letters and speech sounds, between visual and auditory speech, and between other more arbitrary auditory and visual events (such as between tones and circles; Hairston et al., 2005). Alternatively, considering the modifying effects that reading development has at the neural level (Dehaene et al., 2010), the overall diminished activity observed in the dyslexic readers may not be a deficit per se, but a consequence of the failure to establish adequate grapheme-phoneme associations during initial reading development (Blomert, 2011). A more likely interpretation, since differences were found not only in audiovisual speech, but also in its unimodal components (auditory and visual), is that deficits in the processing of the unisensory stimuli result in difficulties with multisensory processing. The current data suggest that the processing of visual speech might be particularly problematic in dyslexia. Typical and dyslexic readers differed in how much they benefited from visual information during audiovisual processing, with dyslexic readers showing reduced activity in the left fusiform gyrus. The fusiform gyrus is involved in audiovisual speech perception (Stevenson et al., 2010; Wyk et al., 2010) and comprehension (McGettigan et al., 2012). The difference found between the groups in the fusiform gyrus is in accordance with DTI studies showing white matter differences between typical and dyslexic readers in this region (e.g., Richards et al., 2008). Furthermore, it is consistent with other fMRI work showing underactivation of the fusiform gyrus in dyslexia (more specifically, in the visual word form area; McCandliss et al., 2003) in response to written words and pseudowords (Helenius et al., 1999; Salmelin et al., 1996; Shaywitz et al., 1998). The visual word form area is a portion of the left fusiform gyrus specifically sensitive to visual word forms and its attributes (McCandliss et al., 2003). Hence, the differences found in the present study between typical and dyslexic readers could reflect differences between the groups in orthographic processing, when perceiving the visual component of audiovisual speech. However, in the present study, the cluster found to be different between typical and dyslexic readers $(x=30, y=-66, z=-12)$ was closer to the clusters more active for facial processing (visual speech $>$ word condition: $x=37 \pm 5, y=-63 \pm 13, z=-16 \pm 2$ in MNI space) than to the clusters more active for word processing (words $>$ visual speech condition; $x=-29 \pm 1, y=-39 \pm 1, z=-14 \pm 2$ in MNI space) and showed increased activity during the visual speech condition, when compared to the auditory speech condition (see Fig. 10). Moreover, the visual speech cluster seems to overlap with the fusiform face area ( $x=41, y=-54, z=-18$; Kanwisher et al., 1997), a portion of the fusiform gyrus involved in detecting and identifying faces (Grill-Spector et al., 2004). The fusiform face area is connected to the superior temporal sulcus (Blank et al., 2011). Its activation is higher when subjects are asked to recognize the speaker than when they are asked to recognize speech (von Kriegstein, 2012). Furthermore, activity in this portion of the fusiform gyrus is positively correlated with face benefits in speaker recognition (von Kriegstein et al., 2008). The differences between typical and dyslexic readers might thus be reflecting differences in face processing. Interestingly, Rüsseler et al. (2015) reported deficient processing of moving faces in dyslexic readers, reflected as a smaller N170 component, which seems to have its neuronal source in the fusiform gyrus (Deffke et al., 2007; Linkenkaer-Hansen et al., 1998; Sadeh et al., 2010). Visual speech processing is a strong and consistent longitudinal correlate of both reading and spelling development in typical children (Kyle and Harris, 2011), possibly supporting the development of the phonological skills needed for reading (Woll, 2012). Thus, if better visual speech skills result in more distinct phonological representations, which in turn help individuals when learning to read, then differences in the benefit taken from visual speech information in language acquisition could underlie differences in reading ability.

The dyslexic readers' decreased activation during audiovisual speech processing might appear to be at odds with evidence showing stronger ventral visual cortex activity for dyslexic readers in the study by Pekkola et al. (2006). In that study, however, stronger ventral visual cortex activity for dyslexic readers was only observed when contrasting incongruent with congruent audiovisual stimuli. It could be the case, therefore, that dyslexic readers make use of different strategies when perceiving congruent and incongruent stimuli (Hayes et al., 2003) and that these differences are somehow reflected in the regions of interest in that study. Additionally, the different results might be explained by differences in the choice of regions of interest: that some areas show decreased activation in dyslexia does not preclude that increased activity is observed in different areas.

Superadditivity during audiovisual processing was found in both heteromodal and unimodal areas, in accordance with what had been previously shown (e.g., Calvert et al., 2000, 2001; Giard and Peronnet, 1999). Again, differences were found between typical and dyslexic readers, with dyslexic readers showing decreased activity in the regions of interest, when compared to the typical readers. Since superadditivity seems to be associated with audiovisual integration, as indicated by the size of the audiovisual benefit (Werner \& Noppeney, 2009), dyslexic readers may have benefited less from audiovisual stimulation than typical readers, as previously shown behaviorally (Ramirez and Mann, 2005; van Laarhoven et al., 2016). 
Finally, some limitations of the present study should be addressed. First, given the limited amount of imaging data and the absence of localizing tasks, we were unable to define functional ROIs for audiovisual processing, and thus had to define the regions anatomically. Averaging over large areas may be problematic and hide effects that otherwise would be observable, particularly in regions with different functions per sub-region, such as the superior temporal gyrus. Second, despite the prevalence of superadditivity measures in the audiovisual literature, its meaningfulness and ideal operationalization are still under debate (James and Stevenson, 2012), with, for instance, the use of different criteria impacting the results found. Even though the measure of superadditivity chosen here is considered to be conservative, the results should be interpreted with the appropriate caution.

\section{Conclusion}

Since reading ability depends on the adequate development of grapheme-phoneme (audiovisual) associations, audiovisual processing difficulties might underlie reading impairment in dyslexia. In the present fMRI study, we tested whether typical and dyslexic adult readers differed in audiovisual speech processing. When compared to typical readers, dyslexic readers: a) performed behaviorally worse in the 1back task when responding to repetitions in the visual speech condition; b) showed less activation in unimodal and heteromodal areas in all speech conditions (auditory, visual, and audiovisual); and c) while processing audiovisual speech, showed less activation in a cluster in the left posterior fusiform gyrus. Taken together, our results suggest the existence of audiovisual speech processing difficulties in dyslexia. They also indicate that such difficulties might result from differences between normal and dyslexic readers in the processing of the unisensory components of speech. More specifically, dyslexic readers seemed to use visual information during audiovisual speech processing differently than typical readers. In addition to earlier work suggesting deficient processing in the fusiform gyrus in response to letter strings (Helenius et al., 1999; Salmelin et al., 1996; Shaywitz et al., 1998), our study adds that there appears to be less activation in the fusiform gyrus in response to visual speech information during audiovisual speech perception in dyslexic readers. Since visual speech processing supports the development of phonological skills that are fundamental in reading, differences in visual speech processing might contribute to differences in reading ability.

\section{Acknowledgements}

MAG was supported by an Innovational Research Incentives Scheme Veni grant (\#275-89-017) from the Netherlands Organisation for Scientific Research. We thank José Marques, Marcel Zwiers, and Paul Gaalman for their technical support and all participants for their cooperation.

\section{Appendix A}

See Table A1

Table A1

List of stimuli per condition.

\begin{tabular}{lll}
\hline Words & Illegal letter strings & Auditory, visual, audiovisual \\
\hline voorbij & qfntp & be \\
zieke & gdbpw & by \\
luilak & szvtf & de \\
trekken & mknrq & do \\
dichten & lxcgd & fu \\
worden & zpqtk & fo \\
roeping & rlwpz & yu \\
zorgvol & tshwm & ye \\
nanacht & xqnjv & fa \\
wetsrol & zfklg & fy \\
morgen & brcpd & ji \\
hamer & pslzf & ju \\
slopen & knhvw & ki \\
bezig & xgrbd & ko \\
hanger & cqtjm & li \\
stamtafel & lsjdw & lu \\
aanplanten & fbkvg & mo \\
steigeren & nxztm & my \\
herkenning & fhcvq & nci \\
bijeenkomst & pbqnw & nu \\
verhuizen & lrxtg & pu \\
uithuilen & kmwpj & py \\
grootmoeder & rzncx & ro \\
heenlopen & sgdlt & ry \\
rekenen & wfgcb & sy \\
navliegen & jskxq & su \\
schoenveter & vtzpl & ty \\
kibbelen & sxbjt & ti \\
frommelen & dgwcl & wu \\
saluutschot & mvfrz & wy \\
\hline & & \\
\hline
\end{tabular}




\section{Appendix B}

see Tables B1-B3

Table B1

Brain regions showing activity during each of the written stimuli conditions, against baseline.

\begin{tabular}{|c|c|c|c|c|c|c|c|c|}
\hline \multirow[t]{2}{*}{ Group } & \multirow[t]{2}{*}{ Condition } & \multirow[t]{2}{*}{ Cluster size } & \multirow[t]{2}{*}{$p_{\text {FWE }}$} & \multicolumn{5}{|l|}{ Local Maxima } \\
\hline & & & & Anatomical area & $\mathbf{x}$ & $\mathbf{y}$ & $\mathbf{z}$ & $\mathbf{t}$ \\
\hline \multirow[t]{33}{*}{ Typical readers } & \multirow[t]{14}{*}{ Words } & \multirow[t]{3}{*}{1241} & \multirow[t]{3}{*}{$\mathrm{p}<.001$} & $\mathrm{R}$ lingual gyrus & 24 & -88 & -8 & 10.93 \\
\hline & & & & $\mathrm{R}$ inferior occipital gyrus & 32 & -92 & 0 & 8.27 \\
\hline & & & & $\mathrm{R}$ calcarine fissure & 24 & -92 & 4 & 8.21 \\
\hline & & 2494 & $\mathrm{p}<.001$ & L inferior occipital gyrus & -20 & -94 & -10 & 10.71 \\
\hline & & 187 & $\mathrm{p}<.001$ & L superior parietal gyrus & -28 & -52 & 52 & 7.75 \\
\hline & & \multirow[t]{2}{*}{413} & \multirow[t]{2}{*}{$\mathrm{p}<.001$} & $\mathrm{R}$ precentral gyrus & 36 & -14 & 64 & 6.68 \\
\hline & & & & $\mathrm{R}$ postcentral gyrus & 52 & -12 & 54 & 5.79 \\
\hline & & \multirow[t]{2}{*}{109} & $\mathrm{p}<.001$ & $\mathrm{~L}$ insula & -32 & 20 & 2 & 6.58 \\
\hline & & & & L IFG - triangular part & -44 & 28 & 0 & 4.07 \\
\hline & & 368 & $\mathrm{p}<.001$ & L precentral gyrus & -48 & 4 & 40 & 5.93 \\
\hline & & & & $\mathrm{L}$ rolandic operculum & -54 & 6 & 12 & 4.75 \\
\hline & & 171 & $\mathrm{p}<.001$ & L supp. motor area & -2 & -2 & 62 & 5.87 \\
\hline & & 301 & $\mathrm{p}<.001$ & $\mathrm{~L}$ inferior parietal & -52 & -28 & 44 & 5.62 \\
\hline & & 161 & $\mathrm{p}<.001$ & L middle temporal gyrus & -46 & -46 & 6 & 5.32 \\
\hline & Illegal letter strings & 2869 & $\mathrm{p}<.001$ & L inferior occipital gyrus & -34 & -86 & -10 & 12.01 \\
\hline & & & & L fusiform gyrus & -42 & -76 & -14 & 10.32 \\
\hline & & 2659 & $\mathrm{p}<.001$ & $\mathrm{R}$ lingual gyrus & 24 & -88 & -8 & 10.82 \\
\hline & & & & $\mathrm{R}$ middle occipital gyrus & 42 & -80 & 2 & 8.04 \\
\hline & & 1644 & $\mathrm{p}<.001$ & L superior parietal gyrus & -30 & -58 & 60 & 8.08 \\
\hline & & & & L inferior parietal gyrus & -46 & -36 & 52 & 7.50 \\
\hline & & 1060 & $\mathrm{p}<.001$ & L precentral gyrus & -46 & 4 & 40 & 7.45 \\
\hline & & 1420 & $\mathrm{p}<.001$ & $\mathrm{R}$ postcentral gyrus & 46 & -24 & 48 & 7.24 \\
\hline & & & & $\mathrm{R}$ inferior parietal gyrus & 50 & -34 & 52 & 6.68 \\
\hline & & & & R superior parietal gyrus & 32 & -58 & 58 & 5.66 \\
\hline & & 698 & $\mathrm{p}<.001$ & L supp. motor area & -2 & 4 & 58 & 7.21 \\
\hline & & 223 & $\mathrm{p}<.001$ & $\mathrm{R}$ hemispheric cerebellum lobule VIII & 16 & -72 & -50 & 6.05 \\
\hline & & & & $\mathrm{R}$ hemispheric cerebellum lobule VIIB & 8 & -74 & -40 & 5.91 \\
\hline & & 146 & $\mathrm{p}<.001$ & $\mathrm{~L}$ insula & -30 & 18 & 6 & 6.04 \\
\hline & & 274 & $\mathrm{p}<.001$ & $\mathrm{R}$ precentral gyrus & 50 & 8 & 38 & 6.04 \\
\hline & & 77 & $\mathrm{p}<.05$ & L hemispheric cerebellum lobule VIII & -24 & -66 & -50 & 5.92 \\
\hline & & 211 & $\mathrm{p}<.001$ & $\mathrm{R}$ insula & 34 & 26 & -2 & 5.32 \\
\hline & & & & R IFG - orbital part & 32 & 26 & -12 & 4.84 \\
\hline & & 84 & $\mathrm{p}<.05$ & L postcentral gyrus & -56 & -18 & 26 & 4.65 \\
\hline Dyslexic readers & Words & 2003 & $\mathrm{p}<.001$ & $\mathrm{R}$ inferior occipital gyrus & 24 & -94 & -8 & 16.18 \\
\hline & & & & $\mathrm{R}$ calcarine fissure & 24 & -90 & 2 & 12.54 \\
\hline & & 2611 & $\mathrm{p}<.001$ & L inferior occipital gyrus & -16 & -96 & -8 & 13.99 \\
\hline & & & & L middle occipital gyrus & -26 & -96 & 0 & 11.58 \\
\hline & & 248 & $\mathrm{p}<.001$ & L precentral gyrus & -50 & 2 & 42 & 6.03 \\
\hline & & & & R IFG - opercular part & -40 & 4 & 30 & 5.52 \\
\hline & & 107 & $\mathrm{p}<.01$ & $\mathrm{R}$ precentral gyrus & 46 & 6 & 34 & 5.80 \\
\hline & & 84 & $\mathrm{p}<.05$ & L supp. motor area & -4 & 8 & 56 & 5.72 \\
\hline & & 85 & $\mathrm{p}<.05$ & L superior parietal gyrus & -30 & -64 & 54 & 5.10 \\
\hline & & 94 & $\mathrm{p}<.01$ & $\mathrm{R}$ angular gyrus & 28 & -60 & 48 & 4.47 \\
\hline & Illegal letter strings & 3149 & $\mathrm{p}<.001$ & $\mathrm{R}$ lingual gyrus & 20 & -90 & -8 & 15.34 \\
\hline & & & & $\mathrm{R}$ inferior occipital gyrus & 26 & -94 & -2 & 11.50 \\
\hline & & & & $\mathrm{R}$ hemispheric cerebellum lobule VI & 8 & -74 & -18 & 10.42 \\
\hline & & 2914 & $\mathrm{p}<.001$ & L inferior occipital gyrus & -20 & -96 & -10 & 12.89 \\
\hline & & & & L middle occipital gyrus & -24 & -94 & 0 & 10.26 \\
\hline & & 420 & $\mathrm{p}<.001$ & L precentral gyrus & -46 & 4 & 36 & 7.59 \\
\hline & & 766 & $\mathrm{p}<.001$ & $\mathrm{R}$ angular gyrus & 30 & -58 & 50 & 7.50 \\
\hline & & & & $\mathrm{R}$ middle occipital gyrus & 30 & -62 & 36 & 6.62 \\
\hline & & 986 & $\mathrm{p}<.001$ & L inferior parietal gyrus & -34 & -50 & 48 & 7.24 \\
\hline & & 449 & $\mathrm{p}<.001$ & R supp. motor area & 2 & 16 & 52 & 6.99 \\
\hline & & & & L supp. motor area & -2 & 8 & 56 & 6.80 \\
\hline & & & & R superior frontal gyrus & 6 & 28 & 44 & 4.32 \\
\hline & & 249 & $\mathrm{p}<.001$ & R precentral gyrus & 42 & 4 & 28 & 6.77 \\
\hline & & & & R IFG - opercular part & 34 & 6 & 30 & 4.50 \\
\hline & & & & $\mathrm{R}$ middle frontal gyrus & 50 & 14 & 48 & 3.75 \\
\hline & & 283 & $\mathrm{p}<.001$ & $\mathrm{R}$ insula & 34 & 20 & 4 & 6.54 \\
\hline & & 285 & $\mathrm{p}<.001$ & R postcentral gyrus & 46 & -28 & 46 & 6.20 \\
\hline & & 87 & $\mathrm{p}<.05$ & R IFG - triangular part & 48 & 36 & 28 & 6.00 \\
\hline & & 86 & $\mathrm{p}<.05$ & R IFG - orbital part & 44 & 48 & -12 & 5.49 \\
\hline & & 75 & $\mathrm{p}<.05$ & $\mathrm{~L}$ insula & -34 & 20 & 2 & 5.12 \\
\hline
\end{tabular}

R: right, L: left, IFG: inferior frontal gyrus, $\mathrm{P}_{\mathrm{FWE}}$ : family-wise corrected $p$. 
Table B2

Brain regions showing activity during each of the audiovisual speech conditions, against baseline.

\begin{tabular}{|c|c|c|c|c|c|c|c|c|}
\hline \multirow[t]{2}{*}{ Group } & \multirow[t]{2}{*}{ Condition } & \multirow[t]{2}{*}{ Cluster size } & \multirow[t]{2}{*}{$p_{\text {FWE }}$} & \multicolumn{5}{|l|}{ Local Maxima } \\
\hline & & & & Anatomical area & $\mathbf{x}$ & $\mathbf{y}$ & $\mathbf{z}$ & $\mathbf{t}$ \\
\hline \multirow[t]{53}{*}{ Typical readers } & Auditory & 3066 & $\mathrm{p}<.001$ & L superior temporal gyrus & -64 & -18 & 8 & 12.64 \\
\hline & & & & L middle temporal gyrus & -54 & -36 & 10 & 12.12 \\
\hline & & 3321 & $\mathrm{p}<.001$ & $\mathrm{R}$ middle temporal gyrus & 56 & -28 & 2 & 12.15 \\
\hline & & & & $\mathrm{R}$ superior temporal gyrus & 64 & -28 & 4 & 10.84 \\
\hline & & 292 & $\mathrm{p}<.001$ & L hemispheric cerebellum lobule VI & -28 & -62 & -24 & 10.46 \\
\hline & & & & L cerebellum crus I & -40 & -70 & -26 & 4.67 \\
\hline & & 691 & $\mathrm{p}<.001$ & L supp. motor area & -4 & 4 & 58 & 9.16 \\
\hline & & & & $\mathrm{R}$ supp. motor area & 6 & 2 & 66 & 6.20 \\
\hline & & 405 & $\mathrm{p}<.001$ & $\mathrm{R}$ hemispheric cerebellum lobule VI & 32 & -62 & -26 & 7.61 \\
\hline & & & & $\mathrm{R}$ cerebellum crus II & 16 & -76 & -36 & 6.10 \\
\hline & & & & $\mathrm{R}$ hemispheric cerebellum lobule VIII & 22 & -70 & -48 & 5.35 \\
\hline & & 194 & $\mathrm{p}<.001$ & L hemispheric cerebellum lobule VIIB & -14 & -78 & -44 & 7.14 \\
\hline & & & & L hemispheric cerebellum lobule VIII & -30 & -66 & -50 & 5.70 \\
\hline & & & & L cerebellum crus II & -12 & -76 & -34 & 5.45 \\
\hline & & 294 & $\mathrm{p}<.001$ & $\mathrm{~L}$ insula & -34 & 20 & 2 & 6.57 \\
\hline & & 951 & $\mathrm{p}<.001$ & R IFG - opercular part & 50 & 8 & 22 & 6.34 \\
\hline & & & & $\mathrm{R}$ insula & 32 & 26 & -2 & 5.95 \\
\hline & & & & $\mathrm{R}$ precentral gyrus & 44 & 2 & 44 & 5.54 \\
\hline & & 388 & $\mathrm{p}<.001$ & L IFG - opercular part & -58 & 8 & 14 & 6.26 \\
\hline & & & & L precentral gyrus & -44 & 0 & 26 & 5.20 \\
\hline & & 354 & $\mathrm{p}<.001$ & $\mathrm{R}$ inferior parietal & 48 & -52 & 58 & 5.91 \\
\hline & & 213 & $\mathrm{p}<.001$ & $\mathrm{R}$ middle frontal gyrus & 42 & 36 & 34 & 5.05 \\
\hline & & 86 & $\mathrm{p}<.05$ & L middle frontal gyrus & -36 & 48 & 8 & 4.96 \\
\hline & & & & L IFG - triangular part & -36 & 38 & 14 & 4.18 \\
\hline & Visual & 4710 & $\mathrm{p}<.001$ & $\mathrm{R}$ insula & 36 & 26 & 0 & 12.50 \\
\hline & & & & R IFG - opercular part & 52 & 18 & 6 & 11.93 \\
\hline & & 3359 & $\mathrm{p}<.001$ & L insula & -34 & 20 & 4 & 12.31 \\
\hline & & & & L IFG - orbital part & -44 & 44 & -4 & 9.01 \\
\hline & & & & L precentral gyrus & -44 & -2 & 52 & 8.97 \\
\hline & & 10459 & $\mathrm{p}<.001$ & L crus I & -38 & -66 & -24 & 11.80 \\
\hline & & & & $\mathrm{R}$ fusiform gyrus & 40 & -52 & -18 & 11.17 \\
\hline & & & & $\mathrm{R}$ middle occipital gyrus & 32 & -90 & 16 & 11.06 \\
\hline & & 1902 & $\mathrm{p}<.001$ & R supp. motor area & 6 & 24 & 50 & 10.37 \\
\hline & & & & L superior frontal gyrus & -8 & 20 & 44 & 9.90 \\
\hline & & & & L supp. motor area & -2 & 18 & 52 & 9.32 \\
\hline & & 1546 & $\mathrm{p}<.001$ & $\mathrm{R}$ inferior parietal & 42 & -44 & 50 & 9.60 \\
\hline & & 1442 & $\mathrm{p}<.001$ & $\mathrm{~L}$ inferior parietal & -54 & -38 & 50 & 8.29 \\
\hline & Audiovisual & 10051 & $\mathrm{p}<.001$ & $\mathrm{R}$ middle temporal gyrus & 56 & -28 & 2 & 13.76 \\
\hline & & & & R superior temporal gyrus & 60 & -12 & -6 & 12.23 \\
\hline & & 3343 & $\mathrm{p}<.001$ & L superior temporal gyrus & -58 & -18 & 6 & 11.38 \\
\hline & & & & L middle temporal gyrus & -54 & -40 & 12 & 9.63 \\
\hline & & 1223 & $\mathrm{p}<.001$ & $\mathrm{~L}$ insula & -36 & 20 & 0 & 8.87 \\
\hline & & & & L precentral gyrus & -44 & -2 & 52 & 8.12 \\
\hline & & 1722 & $\mathrm{p}<.001$ & $\mathrm{R}$ precentral gyrus & 48 & 2 & 46 & 8.63 \\
\hline & & & & R IFG - opercular part & 54 & 18 & 20 & 8.09 \\
\hline & & 410 & $\mathrm{p}<.001$ & $\mathrm{R}$ insula & 34 & 26 & 0 & 7.69 \\
\hline & & & & R IFG - opercular part & 50 & 20 & -2 & 4.54 \\
\hline & & 521 & $\mathrm{p}<.001$ & R supp. motor area & 4 & 4 & 66 & 6.91 \\
\hline & & & & L supp. motor area & -6 & 4 & 58 & 6.09 \\
\hline & & 219 & $\mathrm{p}<.001$ & L middle frontal gyrus & -38 & 48 & 8 & 6.82 \\
\hline & & 339 & $\mathrm{p}<.001$ & $\mathrm{R}$ inferior parietal & 42 & -50 & 56 & 5.48 \\
\hline & & & & $\mathrm{R}$ angular gyrus & 52 & -58 & 52 & 4.47 \\
\hline & & 113 & $\mathrm{p}<.01$ & $\mathrm{~L}$ inferior parietal & -32 & -58 & 50 & 4.77 \\
\hline
\end{tabular}


Table B2 (continued)

\begin{tabular}{|c|c|c|c|c|c|c|c|c|}
\hline \multirow[t]{2}{*}{ Group } & \multirow[t]{2}{*}{ Condition } & \multirow[t]{2}{*}{ Cluster size } & \multirow[t]{2}{*}{$p_{\text {FWE }}$} & \multicolumn{5}{|l|}{ Local Maxima } \\
\hline & & & & Anatomical area & $\mathbf{x}$ & $\mathbf{y}$ & $\mathbf{z}$ & $\mathbf{t}$ \\
\hline \multirow[t]{49}{*}{ Dyslexic readers } & Auditory & 5910 & $\mathrm{p}<.001$ & R superior temporal gyrus & 62 & -24 & 10 & 18.75 \\
\hline & & 4841 & $\mathrm{p}<.001$ & L superior temporal gyrus & -58 & -24 & 6 & 13.93 \\
\hline & & & & L middle temporal gyrus & -62 & -12 & 0 & 12.11 \\
\hline & & 1002 & $\mathrm{p}<.001$ & $\mathrm{R}$ hemispheric cerebellum lobule VI & 28 & -62 & -24 & 8.22 \\
\hline & & & & $\mathrm{R}$ cerebellum crus II & 12 & -78 & -36 & 7.63 \\
\hline & & & & $\mathrm{R}$ cerebellum crus I & 40 & -70 & -24 & 7.62 \\
\hline & & 949 & $\mathrm{p}<.001$ & L cerebellum crus I & -32 & -58 & -32 & 7.79 \\
\hline & & & & $\mathrm{R}$ hemispheric cerebellum lobule VIIB & -22 & -74 & -42 & 7.30 \\
\hline & & & & L cerebellum crus II & -10 & -82 & -34 & 6.30 \\
\hline & & 1126 & $\mathrm{p}<.001$ & R supp. motor area & 4 & 8 & 60 & 7.30 \\
\hline & & 373 & $\mathrm{p}<.001$ & $\mathrm{R}$ inferior parietal & 38 & -48 & 42 & 6.06 \\
\hline & & & & R supramarginal gyrus & 54 & -28 & 50 & 5.00 \\
\hline & & 134 & $\mathrm{p}<.001$ & L middle occipital gyrus & -28 & -56 & 38 & 4.60 \\
\hline & & & & L inferior parietal & -38 & -52 & 46 & 4.33 \\
\hline & & 95 & $\mathrm{p}<.01$ & L precentral gyrus & -46 & -4 & 54 & 5.42 \\
\hline & & & & L middle frontal gyrus & -32 & 0 & 54 & 4.70 \\
\hline & Visual & 11614 & $\mathrm{p}<.001$ & $\mathrm{R}$ hemispheric cerebellum lobule VI & 30 & -60 & -26 & 13.56 \\
\hline & & & & L calcarine fissure & -14 & -100 & 0 & 12.47 \\
\hline & & 2086 & $\mathrm{p}<.001$ & L insula & -34 & 18 & -2 & 13.53 \\
\hline & & & & L precentral gyrus & -44 & -4 & 50 & 7.91 \\
\hline & & 4535 & $\mathrm{p}<.001$ & $\mathrm{R}$ insula & 34 & 22 & 2 & 13.41 \\
\hline & & & & R IFG - opercular part & 44 & 10 & 30 & 10.31 \\
\hline & & 2363 & $\mathrm{p}<.001$ & R supp. motor area & 6 & 18 & 50 & 10.78 \\
\hline & & & & R superior frontal gyrus & 6 & 24 & 44 & 8.61 \\
\hline & & 1198 & $\mathrm{p}<.001$ & $\mathrm{R}$ angular gyrus & 36 & -54 & 50 & 8.82 \\
\hline & & & & $\mathrm{R}$ inferior parietal & 32 & -50 & 44 & 7.32 \\
\hline & & 500 & $\mathrm{p}<.001$ & L inferior parietal & -38 & -52 & 52 & 5.74 \\
\hline & & & & L superior parietal gyrus & -32 & -62 & 48 & 5.33 \\
\hline & & 261 & $\mathrm{p}<.001$ & L middle frontal gyrus & -38 & 58 & 2 & 5.42 \\
\hline & Audiovisual & 7595 & $\mathrm{p}<.001$ & R superior temporal gyrus & 60 & -22 & 10 & 15.88 \\
\hline & & & & $\mathrm{R}$ calcarine fissure & 16 & -98 & 0 & 13.77 \\
\hline & & & & $\mathrm{R}$ rolandic operculum & 48 & -20 & 12 & 13.56 \\
\hline & & 7316 & $\mathrm{p}<.001$ & L superior temporal gyrus & -56 & -24 & 6 & 13.75 \\
\hline & & & & L calcarine fissure & -14 & -100 & 0 & 12.18 \\
\hline & & 262 & $\mathrm{p}<.001$ & L insula & -32 & 22 & 4 & 9.52 \\
\hline & & 321 & $\mathrm{p}<.001$ & $\mathrm{R}$ insula & 38 & 22 & -4 & 8.74 \\
\hline & & & & R IFG - triangular part & 48 & 22 & 2 & 4.91 \\
\hline & & 1879 & $\mathrm{p}<.001$ & R IFG - opercular part & 44 & 10 & 30 & 7.84 \\
\hline & & & & $\mathrm{R}$ precentral gyrus & 44 & 8 & 42 & 7.15 \\
\hline & & 866 & $\mathrm{p}<.001$ & L IFG - opercular part & -40 & 10 & 26 & 7.65 \\
\hline & & & & L IFG - triangular part & -52 & 20 & 32 & 6.21 \\
\hline & & 772 & $\mathrm{p}<.001$ & L supp. motor area & -4 & 10 & 54 & 7.19 \\
\hline & & & & R supp. motor area & 8 & 14 & 52 & 6.04 \\
\hline & & 242 & $\mathrm{p}<.001$ & L precentral gyrus & -46 & -2 & 54 & 7.18 \\
\hline & & 233 & $\mathrm{p}<.001$ & $\mathrm{R}$ inferior parietal & 44 & -50 & 52 & 6.72 \\
\hline & & & & $\mathrm{R}$ angular gyrus & 44 & -58 & 50 & 5.45 \\
\hline & & & & $\mathrm{R}$ superior parietal gyrus & 38 & -58 & 56 & 4.49 \\
\hline & & 128 & $\mathrm{p}<.01$ & $\mathrm{~L}$ inferior parietal & -32 & -56 & 44 & 5.63 \\
\hline & & 94 & $\mathrm{p}<.01$ & L middle frontal gyrus & -32 & 44 & 12 & 4.24 \\
\hline
\end{tabular}

R: right, L: left, IFG: inferior frontal gyrus, supp: supplementary, $\mathrm{P}_{\mathrm{FWE}}$ : family-wise corrected $p$.

Table B3

Brain regions showing activity for the superadditivity contrast $(A V>A+V)$.

\begin{tabular}{|c|c|c|c|c|c|c|c|}
\hline \multirow[t]{2}{*}{ Group } & \multirow[t]{2}{*}{ Cluster size } & \multirow[t]{2}{*}{$p_{\text {FWE }}$} & \multicolumn{5}{|l|}{ Local Maxima } \\
\hline & & & Anatomical area & $\mathbf{x}$ & $\mathbf{y}$ & $\mathbf{z}$ & $\mathbf{t}$ \\
\hline \multirow[t]{6}{*}{ Typical readers } & 7660 & $\mathrm{p}<.001$ & L cerebellum crus I & -30 & -82 & -18 & 11.69 \\
\hline & & & $\mathrm{R}$ lingual gyrus & 16 & -88 & -8 & 11.34 \\
\hline & & & L fusiform gyrus & -36 & -62 & -12 & 10.41 \\
\hline & 2545 & $\mathrm{p}<.001$ & R superior temporal sulcus & 66 & -32 & 6 & 9.99 \\
\hline & 141 & $\mathrm{p}<.001$ & L posterior cingulate gyrus & -6 & -48 & 20 & 5.32 \\
\hline & & & L precuneus & -10 & -58 & 20 & 4.84 \\
\hline \multirow[t]{8}{*}{ Dyslexic readers } & 1837 & $\mathrm{p}<.001$ & L middle occipital gyrus & -18 & -98 & 4 & 11.75 \\
\hline & & & L inferior occipital gyrus & -40 & -80 & -10 & 10.55 \\
\hline & & & $\mathrm{L}$ calcarine fissure & -8 & -100 & 4 & 9.67 \\
\hline & 2070 & $\mathrm{p}<.001$ & L superior temporal sulcus & -52 & -22 & 8 & 11.02 \\
\hline & 2289 & $\mathrm{p}<.001$ & $\mathrm{R}$ calcarine fissure & 14 & -100 & 0 & 10.17 \\
\hline & & & $\mathrm{R}$ middle occipital gyrus & 32 & -88 & 8 & 9.07 \\
\hline & & & $\mathrm{R}$ middle temporal gyrus & 44 & -66 & 4 & 8.48 \\
\hline & 1968 & $\mathrm{p}<.001$ & R superior temporal sulcus & 60 & -22 & 10 & 9.58 \\
\hline
\end{tabular}

$\mathrm{R}$ : right, L: left, $\mathrm{P}_{\mathrm{FWE}}$ : family-wise corrected $p$. 


\section{References}

Baart, M., de Boer-Schellekens, L., Vroomen, J., 2012. Lipread-induced phonetic recalibration in dyslexia. Acta Psychol. 140, 91-95.

Barr, D.J., Levy, R., Scheepers, C., Tily, H.J., 2013. Random effects structure for confirmatory hypothesis testing: keep it maximal. J. Mem. Lang. 68 (3), 255-278.

Barraclough, N.E., Xiao, D., Baker, C.I., Oram, M.W., Perret, D.I., 2005. Integration of visual and auditory information by superior temporal sulcus neurons responsive to the sight of actions. J. Cogn. Neurosci. 17, 377-391.

Bates, D., Maechler, M., Bolker, B., Walker, S., 2014. lme4: Linear mixed-effects models using Eigen and S4. R package version 1.1-7. URL: 〈http://CRAN.R-project.org/ package $=$ lme4>.

Beauchamp, M.S., 2005. Statistical criteria in fMRI studies of multisensory integration. Neuroinformatics 3, 93-113.

Beauchamp, M.S., Argall, B.D., Bodurka, J., Duyn, J.H., Martin, A., 2004a. Unraveling multisensory integration: patchy organization within human STS multisensory cortex. Nat. Neurosci. 7 (11), 1190-1192.

Beauchamp, M.S., Lee, K.E., Argall, B.D., Martin, A., 2004b. Integration of auditory and visual information about objects in superior temporal sulcus. Neuron 41, 809-823.

Beauchamp, M.S., Nath, A.R., Pasalar, S., 2010. fMRI-guided transcranial magnetic stimulation reveals that the superior temporal sulcus is a cortical locus of the McGurk effect. J. Neurosci. 30 (7), 2414-2417.

Bekebrede, J., van der Leij, A., Plakas, A., Share, D., Morfidi, E., 2010. Dutch dyslexia in adulthood: core features and variety. Sci. Stud. Read. 14 (2), 183-210.

Blank, H., Anwander, A., von Kriegstein, K., 2011. Direct structural connections between voice-and face-recognition areas. J. Neurosci. 31 (36), 12906-12915.

Blau, V., van Atteveldt, N., Ekkebus, M., Goebel, R., Blomert, L., 2009. Reduced neural integration of letters and speech sounds links phonological and reading deficits in adult dyslexia. Curr. Biol. 19 (6), 503-508.

Blau, V., Reithler, J., van Atteveldt, N., Seitz, J., Gerretsen, P., Goebel, R., Blomert, L., 2010. Deviant processing of letters and speech sounds as proximate cause of reading failure: a functional magnetic resonance imaging study of dyslexic children. Brain 133 (3), 868-879.

Blomert, L., 2006. Protocol Dyslexie Diagnostiek en Behandeling. CvZ, Diemen.

Blomert, L., 2011. The neural signature of orthographic-phonological binding in successful and failing reading development. NeuroImage 57 (3), 695-703.

Brett, M., Anton, J.L., Valabregue, R., Poline, J.B., 2002. Region of interest analysis using the MarsBar toolbox for SPM 99. NeuroImage 16 (2), S497.

Brus, B.T., Voeten, M.J.M., 1999. Eén-minuut-test: vorm A en B: verantwoording en handleiding: schoolvorderingentest voor de technische leesvaardigheid, bestemd voor groep 4 tot en met 8 van het basisonderwijs. Swets \& Zeitlinger.

Bushara, K.O., Grafman, J., Hallett, M., 2001. Neural correlates of auditory-visual stimulus onset asynchrony detection. J. Neurosci. 21 (1), 300-304.

Callan, D.E., Jones, J.A., Munhall, K., Callan, A.M., Kroos, C., Vatikiotis-Bateson, E., 2003. Neural processes underlying perceptual enhancement by visual speech gestures. Neuroreport 14 (17), 2213-2218.

Calvert, G.A., Brammer, M.J., Bullmore, E.T., Campbell, R., Iversen, S.D., David, A.S., 1999. Response amplification in sensory-specific cortices during crossmodal binding. Neuroreport 10 (12), 2619-2623.

Calvert, G.A., Campbell, R., 2003. Reading speech from still and moving faces: the neural substrates of visible speech. J. Cogn. Neurosci. 15 (1), 57-70.

Calvert, G.A., Campbell, R., Brammer, M.J., 2000. Evidence from functional magnetic resonance imaging of crossmodal binding in the human heteromodal cortex. Curr. Biol. 10 (11), 649-657.

Calvert, G.A., Hansen, P.C., Iversen, S.D., Brammer, M.J., 2001. Detection of audio-visual integration sites in humans by application of electrophysiological criteria to the BOLD effect. Neuroimage 14 (2), 427-438.

Caplan, D., Gow, D., Makris, N., 1995. Analysis of lesions by MRI in stroke patients with acoustic-phonetic processing deficits. Neurology 45 (2), 293-298.

Christopher, M.E., Miyake, A., Keenan, J.M., Pennington, B., DeFries, J.C., Wadsworth, S.J., Olson, R.K., 2012. Predicting word reading and comprehension with executive function and speed measures across development: a latent variable analysis. J. Exp. Psychol.: Gen. 141 (3), 470.

de Gelder, B., Vroomen, J., 1998. Impaired speech perception in poor readers: evidence from hearing and speech reading. Brain Lang. 64, 269-281.

de Pessemier, P., Andries, C., 2009. Test voor Gevorderd Lezen en Schrijven. Garant, Antwerpen, Belgium-Apeldoorn, The Netherlands.

Deffke, I., Sander, T., Heidenreich, J., Sommer, W., Curio, G., Trahms, L., Lueschow, A., 2007. MEG/EEG sources of the 170 -ms response to faces are co-localized in the fusiform gyrus. NeuroImage 35 (4), 1495-1501.

Dehaene, S., Pegado, F., Braga, L.W., Ventura, P., Nunes Filho, G., Jobert, A., Cohen, L., 2010. How learning to read changes the cortical networks for vision and language. Science 330 (6009), 1359-1364.

Ehri, L.C., 1998. Grapheme-phoneme knowledge is essential for learning to read words in English. In: Metsala, J.L., Ehri, L.C. (Eds.), Word Recognition in Beginning Literacy. Erlbaum, Mahwah, NJ, pp. 3-40.

Elbro, C., Nielsen, I., Petersen, D.K., 1994. Dyslexia in adults: evidence for deficits in nonword reading and in the phonological representation of lexical items. Ann. Dyslexia 44 (1), 203-226.

Francisco, A.A., Jesse, A., Groen, M.A., McQueen, J.M., 2017. A general audiovisual temporal processing deficit in adult readers with dyslexia. J. Speech Lang. Hear. Res. 60, 144-158.

Gabrieli, J.D., 2009. Dyslexia: a new synergy between education and cognitive neuroscience. Science 325 (5938), 280-283.

Giard, M.H., Peronnet, F., 1999. Auditory-visual integration during multimodal object recognition in humans: a behavioral and electrophysiological study. J. Cogn. Neurosci. 11 (5), 473-490.

Grill-Spector, K., Knouf, N., Kanwisher, N., 2004. The fusiform face area subserves face perception, not generic within-category identification. Nat. Neurosci. 7, 555-562.

Groen, M.A., Jesse, A., 2013. Audiovisual speech perception in children and adoles- cents with developmental dyslexia: no deficit with McGurk stimuli. In: Ouni, S., Berthommier, F., Jesse, A. (Eds.), Proceedings of the International Conference On Audio-visual Speech Processing (pp. 77-80.

Hahn, N., Foxe, J.J., Molholm, S., 2014. Impairments of multisensory integration and cross-sensory learning as pathways to dyslexia. Neurosci. Biobehav. Rev. 47, 384-392.

Hairston, W.D., Burdette, J.H., Flowers, D.L., Wood, F.B., Wallace, M.T., 2005. Altered temporal profile of visual-auditory multisensory interactions in dyslexia. Exp. Brain Res. 166 (3-4), 474-480.

Hayasaka, S., Nichols, T.E., 2003. Validating cluster size inference: random field and permutation methods. NeuroImage 20 (4), 2343-2356.

Hayes, E.A., Tiippana, K., Nicol, T.G., Sams, M., Kraus, N., 2003. Integration of heard and seen speech: a factor in learning disabilities in children. Neurosci. Lett. 351 (1) 46-50.

Helenius, P., Tarkiainen, A., Cornelissen, P., Hansen, P.C., Salmelin, R., 1999. Dissociation of normal feature analysis and deficient processing of letter-strings in dyslexic adults. Cereb. Cortex 9 (5), 476-483.

James, T.W., Stevenson, R.A., 2012. The use of fMRI to assess multisensory integration. In: Wallace, M., Murray, M. (Eds.), Frontiers in the Neural Bases of Multisensory Processes. Taylor and Francis Group, London.

Kanwisher, N., McDermott, J., Chun, M.M., 1997. The fusiform face area: a module in human extrastriate cortex specialized for face perception. J. Neurosci. 17 (11), 4302-4311.

Karipidis, I., Pleisch, G., Röthlisberger, M., Hofstetter, C., Dornbierer, D., Stämpfli, P., Brem, S., 2017. Neural initialization of audiovisual integration in prereaders at varying risk for developmental dyslexia. Hum. Brain Mapp. 38 (2), 1038-1055.

Kast, M., Bezzola, L., Jäncke, L., Meyer, M., 2011. Multi-and unisensory decoding of words and nonwords result in differential brain responses in dyslexic and nondyslexic adults. Brain Lang. 119 (3), 136-148.

Klucharev, V., Möttönen, R., Sams, M., 2003. Electrophysiological indicators of phonetic and non-phonetic multisensory interactions during audiovisual speech perception. Cogn. Brain Res. 18 (1), 65-75.

Kronschnabel, J., Brem, S., Maurer, U., Brandeis, D., 2014. The level of audiovisual print-speech integration deficits in dyslexia. Neuropsychologia 62, 245-261.

Kyle, F.E., Harris, M., 2011. Longitudinal patterns of emerging literacy in beginning deaf and hearing readers. J. Deaf Stud. Deaf Educ. 16 (3), 289-304.

Laurienti, P.J., Perrault, T.J., Stanford, T.R., Wallace, M.T., Stein, B.E., 2005. On the use of superadditivity as a metric for characterizing multisensory integration in functional neuroimaging studies. Exp. Brain Res. 166, 735-769.

Linkenkaer-Hansen, K., Palva, J.M., Sams, M., Hietanen, J.K., Aronen, H.J., Ilmoniemi, R.J., 1998. Face-selective processing in human extrastriate cortex around $120 \mathrm{~ms}$ after stimulus onset revealed by magneto-and electroencephalography. Neurosci. Lett. 253 (3), 147-150.

Macaluso, E., Frith, C.D., Driver, J., 2000. Modulation of human visual cortex by crossmodal spatial attention. Science 289 (5482), 1206-1208.

Macaluso, E., George, N., Dolan, R., Spence, C., Driver, J., 2004. Spatial and temporal factors during processing of audiovisual speech: a PET study. NeuroImage 21 (2), $725-732$.

Makris, N., Goldstein, J.M., Kennedy, D., Hodge, S.M., Caviness, V.S., Faraone, S.V., Seidman, L.J., 2006. Decreased volume of left and total anterior insular lobule in schizophrenia. Schizophr. Res. 83 (2), 155-171.

Maldjian, J.A., Laurienti, P.J., Burdette, J.H., 2004. Precentral gyrus discrepancy in electronic versions of the Talairach atlas. NeuroImage 21 (1), 450-455.

Maldjian, J.A., Laurienti, P.J., Kraft, R.A., Burdette, J.H., 2003. An automated method for neuroanatomic and cytoarchitectonic atlas-based interrogation of fMRI data sets. NeuroImage 19 (3), 1233-1239.

Martin, A., Kronbichler, M., Richlan, F., 2016. Dyslexic brain activation abnormalities in deep and shallow orthographies: a meta-analysis of 28 functional neuroimaging studies. Hum. Brain Mapp. Adv. Online Publ. https://doi.org/10.1002/hbm.23202.

McCandliss, B.D., Cohen, L., Dehaene, S., 2003. The visual word form area: expertise for reading in the fusiform gyrus. Trends Cogn. Sci. 7 (7), 293-299.

McGettigan, C., Faulkner, A., Altarelli, I., Obleser, J., Baverstock, H., Scott, S.K., 2012. Speech comprehension aided by multiple modalities: behavioural and neural interactions. Neuropsychologia 50 (5), 762-776.

Meredith, M.A., Stein, B.E., 1983. Interactions among converging sensory inputs in the superior colliculus. Science 221, 389-391.

Meredith, M.A., Stein, B.E., 1986. Spatial factors determine the activity of multisensory neurons in cat superior colliculus. Brain Res. 365, 350-354.

Molholm, S., Ritter, W., Javitt, D.C., Foxe, J.J., 2004. Multisensory visual-auditory object recognition in humans: a high-density electrical mapping study. Cereb. Cortex 14 (4), 452-465.

Monzalvo, K., Fluss, J., Billard, C., Dehaene, S., Dehaene-Lambertz, G., 2012. Cortical networks for vision and language in dyslexic and normal children of variable socioeconomic status. NeuroImage 61 (1), 258-274.

Möttönen, R., Schürmann, M., Sams, M., 2004. Time course of multisensory interactions during audiovisual speech perception in humans: a magnetoencephalographic study. Neurosci. Lett. 363 (2), 112-115.

Norrix, L.W., Plante, E., Vance, R., 2006. Auditory-visual speech integration by adults with and without language-learning disabilities. J. Commun. Disord. 39 (1), 22-36.

Ojemann, G., Ojemann, J., Lettich, E., R. E. E. G. T, Berger, M., 1989. Cortical language localization in left, dominant hemisphere: an electrical stimulation mapping 
investigation in 117 patients. J. Neurosurg. 71 (3), 316-326.

Paulesu, E., Frith, U., Snowling, M., Gallagher, A., Morton, J., Frackowiak, R.S., Frith, C.D., 1996. Is developmental dyslexia a disconnection syndrome? Brain 119 (1), 143-157.

Pekkola, J., Laasonen, M., Ojanen, V., Autti, T., Jääskeläinen, I.P., Kujala, T., Sams, M., 2006. Perception of matching and conflicting audiovisual speech in dyslexic and fluent readers: an fMRI study at 3 T. NeuroImage 29 (3), 797-807.

Pekkola, J., Ojanen, V., Autti, T., Jääskeläinen, I.P., Möttönen, R., Tarkiainen, A., Sams, M., 2005. Primary auditory cortex activation by visual speech: an fMRI study at $3 \mathrm{~T}$. Neuroreport 16 (2), 125-128.

Penny, W.D., Holmes, A.P., Friston, K., 2003. Random Effects Analysis. Academic Press, San Diego, pp. 843-850.

Ramirez, J., Mann, V., 2005. Using auditory-visual speech to probe the basis of noiseimpaired consonant-vowel perception in dyslexia and auditory neuropathy. J. Acoust. Soc. Am. 118 (2), 1122-1133.

Richards, T., Stevenson, J., Crouch, J., Johnson, L.C., Maravilla, K., Stock, P., Berninger, V., 2008. Tract-based spatial statistics of diffusion tensor imaging in adults with dyslexia. Am. J. Neuroradiol. 29 (6), 1134-1139.

Rosen, S., 2003. Auditory processing in dyslexia and specific language impairment: is there a deficit? What is its nature? Does it explain anything? J. Phon. 31 (3), 509-527.

Rumsey, J.M., Horwitz, B., Donohue, B.C., Nace, K., Maisog, J.M., Andreason, P., 1997. Phonological and orthographic components of word recognition. A PET-rCBF study. Brain 120 (5), 739-759.

Rüsseler, J., Gerth, I., Heldmann, M., Münte, T.F., 2015. Audiovisual perception of natural speech is impaired in adult dyslexics: an ERP study. Neuroscience 287, 55-65.

Sadeh, B., Podlipsky, I., Zhdanov, A., Yovel, G., 2010. Event-related potential and functional MRI measures of face-selectivity are highly correlated: a simultaneous ERP-fMRI investigation. Hum. Brain Mapp. 31 (10), 1490-1501.

Salmelin, R., Kiesilä, P., Uutela, K., Service, E., Salonen, O., 1996. Impaired visual word processing in dyslexia revealed with magnetoencephalography. Ann. Neurol. 40 (2), $157-162$.

Sandak, R., Mencl, W.E., Frost, S.J., Pugh, K.R., 2004. The neurobiological basis of skilled and impaired reading: recent findings and new directions. Sci. Stud. Read. 8 (3), 273-292.

Shaywitz, S.E., Shaywitz, B.A., Fulbright, R.K., Skudlarski, P., Mencl, W.E., Constable, R.T., Lyon, G.R., 2003. Neural systems for compensation and persistence: young adult outcome of childhood reading disability. Biol. Psychiatry 54 (1), 25-33.

Shaywitz, S.E., Shaywitz, B.A., Pugh, K.R., Fulbright, R.K., Constable, R.T., Mencl, W.E., Katz, L., 1998. Functional disruption in the organization of the brain for reading in dyslexia. Proc. Natl. Acad. Sci. USA 95 (5), 2636-2641.

Skipper, J.I., Nusbaum, H.C., Small, S.L., 2005. Listening to talking faces: motor cortical activation during speech perception. NeuroImage 25 (1), 76-89.

Smith, S.M., Jenkinson, M., Woolrich, M.W., Beckmann, C.F., Behrens, T.E., JohansenBerg, H., Niazy, R.K., 2004. Advances in functional and structural MR image analysis and implementation as FSL. Neuroimage 23, S208-S219.

Stevenson, R.A., Altieri, N.A., Kim, S., Pisoni, D.B., James, T.W., 2010. Neural processing of asynchronous audiovisual speech perception. NeuroImage 49 (4), 3308-3318.

Stevenson, R.A., Geoghegan, M.L., James, T.W., 2007. Superadditive BOLD activation in superior temporal sulcus with threshold non-speech objects. Exp. Brain Res. 179, 85-95.

Stevenson, R.A., VanDerKlok, R.M., Pisoni, D.B., James, T.W., 2011. Discrete neural substrates underlie complementary audiovisual speech integration processes. NeuroImage 55 (3), 1339-1345.

Swanson, H.L., Howard, C.B., Saez, L., 2006. Do different components of working memory underlie different subgroups of reading disabilities? J. Learn. Disabil. 39 (3), 252-269.

Swanson, H.L., Zheng, X., Jerman, O., 2009. Working memory, short-term memory, and reading disabilities: a selective meta-analysis of the literature. J. Learn. Disabil. 42 (3), 260-287.

Szycik, G.R., Jansma, H., Münte, T.F., 2009. Audiovisual integration during speech comprehension: an fMRI study comparing ROI-based and whole brain analyses. Hum. Brain Mapp. 30 (7), 1990-1999.

Tzourio-Mazoyer, N., Landeau, B., Papathanassiou, D., Crivello, F., Etard, O., Delcroix, N., Joliot, M., 2002. Automated anatomical labeling of activations in SPM using a macroscopic anatomical parcellation of the MNI MRI single-subject brain. NeuroImage 15 (1), 273-289.

van Bergen, E., de Jong, P.F., Maassen, B., van der Leij, A., 2014. The effect of parents literacy skills and children's preliteracy skills on the risk of dyslexia. J. Abnorm. Child Psychol. 42 (7), 1187-1200.

von Kriegstein, K., 2012. A multisensory perspective on human auditory communication. In: Murray, M.M., Wallace, M.T. (Eds.), The Neural Basis of Multisensory Processes. Taylor \& Francis, Boca Raton, FL, pp. 683-702.

von Kriegstein, K., et al., Dogan, Ö., Grüter, M., Giraud, A.L., Kell, C.A., Grüter, T., 2008. Simulation of talking faces in the human brain improves auditory speech recognition. Proc. Natl. Acad. Sci. USA 105 (18), 6747-6752.

van Laarhoven, T., Keetels, M., Schakel, L., Vroomen, J., 2016. Audio-visual speech in noise perception in dyslexia. Dev. Sci. 21, 1.

Vellutino, F.R., Fletcher, J.M., Snowling, M.J., Scanlon, D.M., 2004. Specific reading disability (dyslexia): what have we learned in the past four decades? J. Child Psychol. Psychiatry 45 (1), 2-40.

Wallace, M.T., Stevenson, R.A., 2014. The construct of the multisensory temporal integration window and its dysregulation in developmental disabilities. Neuropsychologia 64, 105-123.

Werner, S., Noppeney, U., 2010. Superadditive responses in superior temporal sulcus predict audiovisual benefits in object categorization. Cereb. Cortex 20 (8), 1829-1842.

Woll, B., 2012. Speechreading revisited. Deaf. Educ. Int. 14 (1), 16-21.

Woolrich, M.W., Jbabdi, S., Patenaude, B., Chappell, M., Makni, S., Behrens, T., Smith, S.M., 2009. Bayesian analysis of neuroimaging data in FSL. Neuroimage 45 (1), S173-S186.

Wright, T.M., Pelphrey, K.A., Allison, T., McKeown, M.J., McCarthy, G., 2003. Polysensory interactions along lateral temporal regions evoked by audiovisual speech. Cereb. Cortex 13 (10), 1034-1043.

Wyk, B.C.V., Ramsay, G.J., Hudac, C.M., Jones, W., Lin, D., Klin, A., et al., 2010. Cortical integration of audio-visual speech and non-speech stimuli. Brain Cogn. 74, 97-106.

Ye, Z., Rüsseler, J., Gerth, I., Münte, T.F., 2017. Audiovisual speech integration in the superior temporal region is dysfunctional in dyslexia. Neuroscience 365, 1-10. 